\title{
RESPUESTA DE LA ATENCIÓN TEMPRANA ESPAÑOLA A LA SITUACIÓN DE CONFINAMIENTO POR COVID-19
}

\section{Response of the Spanish Early Intervention to the Lockdown Situation due to COVID-19}

\author{
Francisco Alberto García-SÁnchez \\ Universidad de Murcia. Facultad de Educación. Departamento de Métodos de Investigación \\ y Diagnóstico en Educación. Grupo de Investigación en Educación, Diversidad y Calidad \\ fags@um.es
}

Manuel Pacheco Molero

Asociación Española de Intervención en la Primera Infancia (AEIPI)

ASPACE Rioja

Noelia Orcajada Sánchez

Universidad de Murcia. Facultad de Educación. Departamento de Métodos de Investigación y Diagnóstico en Educación. Grupo de Investigación en Educación, Diversidad y Calidad

Recepción: 15 de septiembre de 2020

Aceptación: 13 de enero de 2021

Resumen: La situación extraordinaria de pandemia COVID-19 llevó al confinamiento y a la interrupción de los servicios habituales de Atención Temprana (AT), aunque familias y niños seguían necesitando apoyo. Los profesionales españoles reaccionaron rápidamente, reorganizando la intervención, experimentando con las posibilidades de teleintervención y compartiendo conocimientos y experiencias. Este trabajo revisa los recursos generados, en relación con $\mathrm{AT}$, durante las cinco primeras semanas de confinamiento (desde del 13 de marzo), así como opiniones y sentimientos compartidos por los profesionales. Se identificaron un total de 72 recursos, facilitados a través de redes sociales: documentos (33), videoconferencias y seminarios online (32) y aplicaciones o recursos web (8). Se analizan sus características, temporización, orientación hacia familias o profesionales y su alineación con un paradigma de intervención centrado en la familia o en el niño. Las opiniones y sentimientos de los profesionales se analizan en referencia a los recursos facilitados, las necesidades observadas en las familias, los problemas en la 
teleintervención y ante el planteamiento de vuelta a una 'nueva normalidad'. Los resultados obtenidos complementan la visión de las familias sobre la situación generada por el confinamiento, que ha quedado recogida en otros trabajos. Se concluye sobre la buena salud de la AT en España y la necesidad de profundizar en la implicación proactiva de la familia en el equipo de intervención.

Palabras clave: Atención Temprana; COVID-19; profesionales; teleintervención; formación.

AвstRACT: The extraordinary situation of the COVID-19 pandemic led to the lockdown and interruption of the usual Early Intervention (EI) services, although families and children still needed support. The Spanish professionals reacted quickly, reorganizing the intervention, experimenting with the possibilities of tele-intervention and sharing knowledge and experiences. This work reviews the resources generated, in relation to EI, during the first five weeks of lockdown (from March 13). This work also reviews opinions and feelings that were shared by professionals. 72 resources were identified, provided through social networks: documents (33), videoconferences and online seminars (32) and applications or web resources (8). Its characteristics, timing, orientation to families or professionals, and its alignment with a paradigm of intervention centered on the family or the child are analyzed. The opinions and feelings of the professionals are analyzed in reference to the resources provided, the needs observed in the families, the problems in tele-intervention and in the face of the approach to return to a "new normality". The results obtained complement the vision of families about the situation generated by the lockdown, which has been collected in other works. It concludes on the good health of EI in Spain and the need to deepen the proactive involvement of the family in the intervention team.

KeYwords: Early Childhood Intervention; COVID-19; professionals; tele-intervention; training.

\section{Introducción}

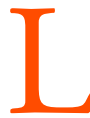

A LLEGADA DE LAS MEDIDAS DE CONFINAMIENTO, sobrevenidas a la expansión de la infección por Covid-19 en España, nos alcanzó a todos de forma inesperada. A pesar de ir conociendo las cifras de infectados en nuestro país, no tuvimos la capacidad de anticiparnos a esta situación. A la cual, además, llegamos acompañados de una cascada de informaciones que apenas daba tiempo a procesar, antes de una nueva noticia aún más alarmante que la anterior. Entre el jueves 12 y el viernes 13 de marzo empezaron a sucederse notificaciones de cierres de diferentes centros que daban atención a las personas con discapacidad a lo largo de su ciclo vital. Así, el viernes 13 ya estaba ordenado el cierre de la mayoría de los centros de Atención Temprana de todo el territorio español, en decisiones acordadas por las diferentes administraciones autonómicas.

A pesar de que el sábado 14 de marzo se promulgara el Real Decreto 463/2020, por el que se declara el estado de alarma para la gestión de la situación de crisis sanitaria 
ocasionada por el COVID-19, la situación inicial en el sector de la Atención Temprana, como en otros muchos, era de total desconcierto. Inicialmente, muchos profesionales fueron apremiados a acudir a sus respectivos puestos de trabajo, los días 16 y 17. Se encargaron de avisar a las familias del cierre del servicio; realizaron tareas de coordinación con el equipo o trabajo personal de despacho, y empezaron a pensar en organizar una continuidad del trabajo, pero sin saber muy bien cómo.

Ante el confinamiento y las limitaciones de movilidad que implicaba, la población se volcó en compartir y comunicarse a través de redes sociales (Acebes y Montanera, 2020; Sánchez, 2020). La tecnología se convirtió en un recurso imprescindible para continuar conectados e incluso trabajando. La Atención Temprana no quedó ajena a este fenómeno y el teletrabajo empezó a generalizarse como la única posibilidad de mantener una intervención que, en esos momentos, no podía realizarse de forma presencial.

Pasados los meses y una vez recuperada la denominada 'nueva normalidad', empezamos a ver los primeros análisis de cómo vivieron las familias atendidas por servicios en Atención Temprana, esas semanas de confinamiento. Distintos trabajos analizaron sus vivencias, el impacto de la situación de confinamiento en sus realidades familiares y la adecuación y satisfacción de los servicios de teleintervención recibidos, tanto a nivel nacional (Martín-Tarrascón y Segarra, 2020; UCCAP y ACAP, 2020) como internacional (Neece et al., 2020).

El objetivo de este trabajo también es analizar el impacto de la situación de confinamiento en la Atención Temprana española, pero desde la perspectiva de los profesionales. Describimos la evolución que siguió la respuesta, en las redes sociales, del conjunto de recursos de Atención Temprana en nuestro país. Para ello, utilizaremos diferentes fondos documentales de organizaciones que, durante las primeras semanas de confinamiento, volcaron esfuerzos para encontrar, facilitar, coordinar y formar a los profesionales y a las familias en las posibilidades de dar continuidad a la intervención de Atención Temprana. Analizaremos, a través de un estudio descriptivo, la secuenciación temporal y los contenidos de los recursos facilitados y los esfuerzos realizados, así como sensaciones y sentimientos expresados por profesionales en su esfuerzo por mantener el contacto y una teleintervención con las familias y los niños.

\section{Panorámica de la Atención Temprana en España en marzo de 2020}

Para entender la respuesta del conjunto de recursos de Atención Temprana en España resulta conveniente recordar la situación en la que se encontraba la disciplina en el momento de la orden de confinamiento.

Aunque con algunas experiencias anteriores, el comienzo de la Atención Temprana en España debemos remontarla a los años 80. Como se señala en la Figura 1, un primer hito, especialmente relevante, debemos fecharlo en el año 2000, con la publicación del Libro Blanco de la disciplina (GAT, 2000). Ciertamente ese texto ya intentaba remarcar la importancia de la familia en la intervención. Pero lo cierto es que seguía manteniendo un modelo de intervención ambulatoria, centrado en las necesidades detectadas por el 
profesional en el niño, heredado de las visiones rehabilitadoras de las décadas iniciales. Por ello, a pesar de su publicación, se seguían detectando carencias en la intervención con la familia, evidenciadas tanto por el propio Grupo de Atención Temprana (GAT, 2011) como por diversos grupos de investigación de universidades españolas. En concreto, en Barcelona, repartidos entre la Universidad Ramon Llul y la Universidad de Barcelona, el equipo del profesor Climent Giné Giné venía publicando trabajos resaltando la necesidad de cambiar hacia una intervención más centrada en la familia (Giné et al., 2009; Giné et al., 2006; Giné et al., 2004; Giné et al., 2005). Igual ocurría en la Universidad Complutense de Madrid, con el equipo de la profesora Pilar Gútiez Cuevas y su grupo de investigación en 'Intervención Temprana: Prevención, Detección e Intervención en el desarrollo y sus alteraciones' (Gútiez, 2010). Y en la Universidad de Murcia, el equipo del profesor Francisco Alberto García-Sánchez, que en 2008 se constituiría como Grupo de Investigación en Educación, Diversidad y Calidad (https:// curie.um.es/curie/catalogo-ficha.duseof_codigo $=1 \&$ perf_codigo $=10 \& \operatorname{cods}=\mathrm{E} 074 \% 08$; www.facebook.com/qdiversidad), trabajaba en la misma línea. Este último defendía un modelo integral de intervención en Atención Temprana, que proponía ir más allá de la intervención ambulatoria, con desplazamientos del profesional al entorno natural, en detrimento del número de sesiones prescritas para el niño. Todo ello con el fin de atender las necesidades y prioridades de la familia en el entorno, aumentar sus competencias, reducir sus fuentes de estrés y mejorar su calidad de vida (Castellanos et al., 2000; Castellanos et al., 2003; García-Sánchez, 2002a, 2002b, 2003; García-Sánchez y Mendieta, 1998; Mendieta y García-Sánchez, 1998).

\section{Figura 1. Evolución histórica de la Atención Temprana en España}

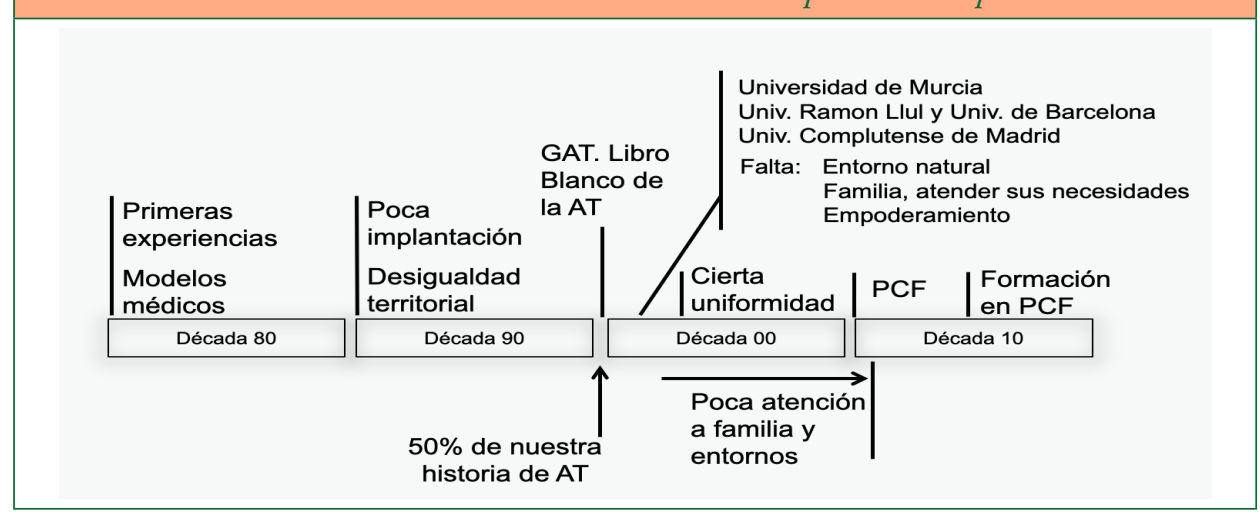

Adaptada de García-Sánchez, F. A. (2018, 28 abril). Atención Temprana centrada en la familia: evolución para la mejora. Ponencia dictada en el II Congreso Nacional de Innovación en Atención Temprana. ASPROMIN, Huelva. Recuperado de http://congresoaspromin.com/ casp/wp-content/uploads/2019/02/01-PO-ATENCIÓN-TEMPRANA-CENTRADA-ENLA-FAMILIA-EVOLUCIÓN-PARA-LA-MEJORA-1.pdf

Con todo, es en la década de 2010 cuando empieza a interiorizarse, entre los profesionales españoles, la idea de que unas prácticas de intervención centradas en la 
familia son algo diferente a unas prácticas ambulatorias centradas en el niño en las que se asesora a las familias desde la perspectiva del profesional (Escorcia et al., 2016; García-Grau et al., 2019; García-Sánchez et al., 2014; Gracia et al., 2019). Y en los últimos cinco años han aparecido las primeras ofertas formativas en estas prácticas de intervención (especialmente en la Universidad de Murcia, en la Universidad Internacional del Mar y en la Universidad Autónoma de Madrid, esta última en colaboración estrecha con Plena inclusión), observándose un claro incremento del interés por esa formación entre los profesionales.

Por todo ello, seguramente el confinamiento llegó en un momento en el que la Atención Temprana en España contaba con importantes fortalezas. Por un lado, con la disponibilidad de una amplia red (siempre mejorable) de recursos y servicios distribuidos por todo el territorio nacional. En ellos se dispone de un volumen importante de profesionales, con distinto grado de veteranía, pero siempre implicados por su alto grado de profesionalidad. Y entre ellos un porcentaje ya significativo de profesionales con alguna formación en prácticas de intervención centradas en la familia, incluso decenas de centros ya transformados o en proceso de transformación a estas prácticas.

\section{Metodología del estudio realizado}

\subsection{Criterios y procedimiento de selección de la información}

Se han utilizado dos fuentes fundamentales de información. Una para identificar los recursos generados, en relación con Atención Temprana, entre el viernes 13 de marzo de 2020 y el viernes 17 de abril de 2020. Este intervalo temporal corresponde a las 5 semanas iniciales tras el anuncio del confinamiento y la promulgación del Real Decreto 463/2020 de 14 de marzo. Otra para identificar sensaciones y sentimientos de los profesionales, ante la necesidad de seguir en contacto con las familias y las dificultades experimentadas para ello, así como de cara ya a la vuelta a la 'nueva normalidad'.

Para identificar los recursos generados en las primeras 5 semanas de confinamiento se han tenido en cuenta, como fuente primordial de información, las notificaciones de publicaciones y eventos que llegaron a la Asociación Española de Intervención en la Primera Infancia (AEIPI). Esta asociación (https://aeipi.org; https://www.facebook. com/groups/AEIPI) surgió en el año 2017, a partir de la iniciativa de profesionales, familias y entidades ilusionadas por la transformación teórico-práctica que venía dándose en la Atención Temprana española desde unos años antes. Una transformación hacia unas prácticas centradas en la familia y desarrolladas fundamentalmente en el entorno natural, internacionalmente recomendadas y basadas en la evidencia científica. Desde su constitución, AEIPI optó por utilizar internet y las redes sociales como vehículo fundamental e indispensable para el contacto de los profesionales y el intercambio de información. Por ello, la situación de confinamiento que se nos imponía no suponía ningún problema para seguir favoreciendo el debate, compartir

Ediciones Universidad de Salamanca / CC BY-NC-ND

Siglo Cero, vol. 52, número extraordinario, 2021, pp. 37-74 
preocupaciones y posibles soluciones. A esta fuente de información hay que añadir la información encontrada a través del rastreo en internet entre las fechas indicadas.

Para identificar las vivencias de los profesionales se utilizaron, como fuente fundamental de información, las ideas expresadas por las 5 profesionales del equipo de intervención centrada en la familia de ASTRAPACE (Murcia), en las sesiones de supervisión reflexiva llevadas a cabo durante la tercera y cuarta semana de marzo, el mes de abril y la primera semana de mayo. Esto permitió, también, registrar sus opiniones ante el planteamiento de vuelta a una 'nueva normalidad'. Este equipo, constituido en 2014, cuenta con 2 psicólogas a tiempo completo y, a tiempo parcial, una logopeda, una fisioterapeuta y una terapeuta ocupacional. Actúan como supervisores 2 profesores de la Universidad de Murcia, miembros del Grupo de Investigación en Educación, Diversidad y Calidad (García-Sánchez, 2017; Tudela et al., 2017). Esta información se completó con opiniones expresadas por otros profesionales de los equipos de intervención ambulatoria de ASTRAPACE y otros centros de la Región de Murcia; y los profesionales de los equipos de intervención centrada en la familia de ASPACE Rioja, del Equipo InSitu de Crevillente, del Equipo SIDI de Málaga, entre otros.

Las sesiones de supervisión reflexiva consisten en reuniones de 3 horas de duración (realizadas por vía telemática durante la etapa del confinamiento), que se llevan a cabo con periodicidad semanal, con la finalidad de generar un espacio para el intercambio de información, pensamientos y sentimientos sobre todo lo que acontece en y alrededor de la intervención centrada en la familia (Parlakian, 2001). Se apoyan, también, en un grupo de WhatsApp, desde el que poder ofrecer reflexión, casi inmediata, ante situaciones surgidas en cualquier momento durante la intervención. De esta forma, la supervisión reflexiva se convierte en un proceso de análisis, con otra persona, de los pensamientos, sentimientos, acciones y reacciones evocadas en el trabajo de estrecha colaboración con los niños pequeños y sus familias (Eggbeer et al., 2008; Mackin-Brown, 2016).

\subsection{Metodología de investigación}

Para analizar los recursos generados, una vez identificados, se procedió a clasificarlos atendiendo a diferentes criterios:

- Tipo de material: videoconferencia (disponible en directo o colgada en alguna plataforma), documentación escrita o aplicación.

- Fecha de aparición, considerando semanas de 1 (del 13 al 19 de marzo) a 5 (del 11 al 17 de abril).

- Entidad responsable.

- Alineación con un paradigma de intervención centrado en la familia o en el niño.

- Naturaleza del contenido abordado:

- Situación en que quedan los centros y servicios.

- Recursos orientados hacia las familias.

- Recursos orientados a los profesionales. 
- Orientado a la formación.

- Plataformas: creadas a raíz de la pandemia o reorientadas para facilitar recursos, la conexión y el intercambio de información.

Para analizar las sensaciones y opiniones de los profesionales, se fueron registrando expresiones aparecidas durante las sesiones de supervisión reflexiva realizadas, así como otras recibidas por los autores del presente trabajo. Para ello, se revisaron las actas de dichas sesiones, así como registros de WhatsApp de diferentes grupos de profesionales de Atención Temprana. A partir de las expresiones registradas, se procedió a su clasificación en diferentes categorías:

- Referidas a los recursos que se iban generando.

- Referidas a las familias, sus necesidades y las interacciones vividas con ellas.

- Referidas a los problemas detectados, desde la perspectiva meramente profesional, a la hora de desarrollar la teleintervención.

\subsection{Medidas}

El estudio realizado es meramente descriptivo, utilizando para ello el análisis cualitativo de los distintos tipos de recursos y registros realizados, a partir del cálculo de estadísticos descriptivos básicos, fundamentalmente frecuencias y porcentajes.

\section{Resultados}

\subsection{Análisis de los recursos generados durante las cinco primeras semanas tras el confinamiento de la población española}

Se identificaron un total de 72 recursos. Todos ellos accesibles, en internet, para profesionales y familias, entre el viernes 13 de marzo de 2020 y el viernes 17 de abril de 2020. Fechas que corresponden a las 5 primeras semanas del confinamiento.

En la Tabla 1 se recogen los 72 recursos identificados. Esta cifra supone un promedio de 2,06 recursos nuevos por día. Se identifican con un número específico y se detalla, para cada uno de ellos, la temática, fecha de publicación, entidad responsable, tipo de documento, detalles de su contenido, dirección url para su consulta e información sobre el alineamiento o no del recurso con un paradigma de intervención concreto. 


\begin{tabular}{|c|c|c|c|c|c|}
\hline \multicolumn{6}{|c|}{ TABLA 1. Descripción y enlaces de los recursos generados (cont.) } \\
\hline N. & $\begin{array}{l}\text { Temá- } \\
\text { tica }\end{array}$ & Fecha & Entidad & Tipo & Detalle y enlaces \\
\hline 1 & $\begin{array}{c}1 \\
\text { Situac. } \\
\text { centros }\end{array}$ & $\begin{array}{l}13 / 3 / \\
2020\end{array}$ & Comunidades & Doc & $\begin{array}{l}\text { Se decreta el cierre la mayoría de los } \\
\text { centros de atención temprana de todo el } \\
\text { territorio español ordenado por las di- } \\
\text { ferentes administraciones autonómicas. } \\
\text { https://plenainclusionmadrid.org/no- } \\
\text { ticias/coronavirus-centos-ocupaciona- } \\
\text { les-y-atencion-temprana/ } \\
\text { Recurso que no se alinea con un para- } \\
\text { digma de intervención específico. }\end{array}$ \\
\hline 2 & $\begin{array}{c}1 \\
\text { Situac. } \\
\text { centros }\end{array}$ & $\begin{array}{l}14 / 3 / \\
2020\end{array}$ & Estado & Doc & $\begin{array}{l}\text { Real Decreto } 463 / 2020 \text {, de } 14 \text { de marzo, } \\
\text { por el que se declara el estado de alarma } \\
\text { para la gestión de la situación de crisis } \\
\text { sanitaria ocasionada por el COVID-19. } \\
\text { h t t p s : / / w w w . b o e e s / e li / e s / } \\
\mathrm{rd} / 2020 / 03 / 14 / 463 / \text { dof/spa/pdf } \\
\text { Recurso que no se alinea con un para- } \\
\text { digma de intervención específico. }\end{array}$ \\
\hline 3 & $\begin{array}{c}1 \\
\text { Situac. } \\
\text { centros }\end{array}$ & $\begin{array}{l}14 / 3 / \\
2020\end{array}$ & AEIPI & Doc & $\begin{array}{l}\text { AEIPI - Lanza las recomendaciones } \\
\text { para la continuidad para los centros a } \\
\text { través de una newsletter y publicando } \\
\text { el contenido en su web } \\
\text { https://drive.google.com/file/d/1UF2z } \\
\text { Gi78tLAgAV1aeyyb355VbRhJmZIg/ } \\
\text { view? usp=sharing } \\
\text { Recurso alineado con el paradigma de } \\
\text { intervención centrado en la familia. }\end{array}$ \\
\hline 4 & $\begin{array}{c}1 \\
\text { Situac. } \\
\text { centros }\end{array}$ & $\begin{array}{l}14 / 3 / \\
2020\end{array}$ & $\begin{array}{l}\text { ACAIT } \\
\text { Málaga }\end{array}$ & Doc & $\begin{array}{l}\text { Plan de apoyo familiar de ACAIT MÁLA- } \\
\text { GA durante el aislamiento por COVID-19. } \\
\text { Desde ACAIT MÁLAGA se realiza una } \\
\text { propuesta a la Junta de Andalucía para } \\
\text { este periodo de suspensión de actividad } \\
\text { al que se han visto obligados los servicios } \\
\text { de Atención Temprana por todo el país } \\
\text { debido al virus COVID-19. } \\
\text { http://acaitmalaga.blogspot. } \\
\text { com/2020/03/plan-de-apoyo-familiar- } \\
\text { de-acait-malaga.html } \\
\text { Recurso que no se alinea con un para- } \\
\text { digma de intervención específico. }\end{array}$ \\
\hline
\end{tabular}




\begin{tabular}{|c|c|c|c|c|c|}
\hline \multicolumn{6}{|c|}{ TABLA 1. Descripción y enlaces de los recursos generados (cont.) } \\
\hline N. & $\begin{array}{c}\text { Temá- } \\
\text { tica }\end{array}$ & Fecha & Entidad & Tipo & Detalle y enlaces \\
\hline $\begin{array}{l}5 \\
\mathrm{a} \\
9\end{array}$ & $\begin{array}{l}4 \text { For- } \\
\text { mación }\end{array}$ & $\begin{array}{l}15 / 3 / \\
2020\end{array}$ & $\begin{array}{l}\text { Doble } \\
\text { Equipo }\end{array}$ & Vídeo & $\begin{array}{l}\text { Doble Equipo: serie de charlas con } \\
\text { profesionales de referencia, sobre te- } \\
\text { mas como resiliencia familiar, partici- } \\
\text { pación en las rutinas diarias, lenguaje } \\
\text { natural asistido e inclusión educativa. } \\
\text { 5. } 15 \text { de marzo: Ideas para estructurar } \\
\text { el tiempo en casa. https://www.fa- } \\
\text { cebook.com/400377880079232/vi- } \\
\text { deos/148997936336404 } \\
\text { 6. } 22 \text { de marzo: Participación en las ru- } \\
\text { tinas diarias. https://www.facebook. } \\
\text { com/4 0037788079232/videos/8 } \\
\text { 77376916020957/ } \\
\text { 7. } 25 \text { de marzo: Resiliencia Familiar. } \\
\text { https://www.facebook.com/40037 } \\
\text { 7880079232/videos/159054428 } \\
\text { 606535/ } \\
\text { 8. 29 de marzo: Lenguaje natural asis- } \\
\text { tido. https://www.facebook.com/40 } \\
\text { 0377880079232/videos/3366658739 } \\
56101 / \text { ?epa=SEARCH_BOX } \\
\text { 9. } 1 \text { de abril: Inclusión La escuela } \\
\text { somos todos. https://www.face- } \\
\text { book.com/40 0377880079232/ } \\
\text { videos/25866 } \\
\text { 17374944592/ } \\
\text { Recursos alineados con el paradig- } \\
\text { ma de intervención centrado en la } \\
\text { familia. }\end{array}$ \\
\hline 10 & $\begin{array}{c}1 \\
\text { Situac. } \\
\text { centros }\end{array}$ & $\begin{array}{c}16 / 3 / \\
2020 \text { y } \\
\text { fechas } \\
\text { poste- } \\
\text { riores }\end{array}$ & $\begin{array}{c}\text { Repartidas } \\
\text { por toda } \\
\text { España }\end{array}$ & Doc & $\begin{array}{l}\text { Muchos centros de atención tem- } \\
\text { prana publican notas de prensa } \\
\text { donde proponen seguir el acompa- } \\
\text { ñamiento y el apoyo a las familias } \\
\text { vía online. } \\
\text { A título de ejemplo: } \\
\text { https://atenciontemprana.org/noti- } \\
\text { cias/covid-19-fundacion-atencion- } \\
\text { temprana-continua-su-atencion- } \\
\text { a-los-ninos-y-familias/ } \\
\text { https://criando247.com/coronavi- } \\
\text { rus-atencion-temprana-online-en- } \\
\text { trevista-a-centro-iria/ } \\
\text { Recurso que no se alinea con un pa- } \\
\text { radigma de intervención específico. }\end{array}$ \\
\hline
\end{tabular}

Ediciones Universidad de Salamanca / CC BY-NC-ND

Siglo Cero, vol. 52, número extraordinario, 2021, pp. 37-74 


\begin{tabular}{|c|c|c|c|c|c|}
\hline \multicolumn{6}{|c|}{ TABLa 1. Descripción y enlaces de los recursos generados (cont.) } \\
\hline N. & $\begin{array}{l}\text { Temá- } \\
\text { tica }\end{array}$ & Fecha & Entidad & Tipo & Detalle y enlaces \\
\hline 11 & $\begin{array}{c}1 \\
\text { Situac. } \\
\text { centros }\end{array}$ & $\begin{array}{l}16 / 3 / \\
2020\end{array}$ & Andalucía & Doc & $\begin{array}{l}\text { Continuidad del proceso de Aten- } \\
\text { ción Infantil Temprana como con- } \\
\text { secuencia de la situación y evolu- } \\
\text { ción del coronavirus (COVID-19). } \\
\text { Orden } 16 \text { de marzo de 2020, por } \\
\text { la que se facilita la continuidad del } \\
\text { proceso de Atención Infantil Tem- } \\
\text { prana como consecuencia de la si- } \\
\text { tuación y evolución del coronavi- } \\
\text { rus (COVID-19) (BOJA de } 20 \text { de } \\
\text { marzo de 2020). Texto completo. } \\
\text { https://www.iustel.com/diario_- } \\
\text { del_derecho/noticia.asp?ref_ius- } \\
\text { tel=1196208 } \\
\text { Recurso que no se alinea con un pa- } \\
\text { radigma de intervención específico. }\end{array}$ \\
\hline 12 & $\begin{array}{c}3 \\
\text { Re- } \\
\text { curs. } \\
\text { profes. }\end{array}$ & $\begin{array}{l}16 / 3 / \\
2020\end{array}$ & ASHA & Doc & $\begin{array}{l}\text { Aparecen recursos internacionales } \\
\text { sobre uso de la telepráctica para } \\
\text { proporcionar servicios de interven- } \\
\text { ción temprana a las familias. } \\
\text { https://blog.asha.org/2020/03/16/ } \\
\text { how-our-early-intervention-prac- } \\
\text { tice-serves-families-through-tele- } \\
\text { practice/ } \\
\text { Recurso alineado con el paradig- } \\
\text { made intervención centrado en la } \\
\text { familia. }\end{array}$ \\
\hline 13 & $\begin{array}{c}5 \\
\text { Plata- } \\
\text { formas }\end{array}$ & $\begin{array}{l}17 / 3 / \\
2020\end{array}$ & $\begin{array}{l}\text { Plena inclu- } \\
\text { sión }\end{array}$ & App & $\begin{array}{l}\text { Plena inclusión lanza la app "Fren- } \\
\text { te al aislamiento. Nos conectamos". } \\
\text { https://www.plenainclusion. } \\
\text { org/informate/actualidad/noti- } \\
\text { cias/2020/plena-inclusion-lanza- } \\
\text { la-app-frente-al-aislamiento-nos- } \\
\text { conectamos } \\
\text { Recurso que no se alinea con un pa- } \\
\text { radigma de intervención específico. }\end{array}$ \\
\hline
\end{tabular}




\begin{tabular}{|c|c|c|c|c|c|}
\hline \multicolumn{6}{|c|}{ TABLa 1. Descripción y enlaces de los recursos generados (cont.) } \\
\hline N. & $\begin{array}{c}\text { Temá- } \\
\text { tica }\end{array}$ & Fecha & Entidad & Tipo & Detalle y enlaces \\
\hline 14 & $\begin{array}{c}2 \\
\text { Rec. } \\
\text { familia }\end{array}$ & $\begin{array}{l}19 / 3 / \\
2020\end{array}$ & AEIPI & Vídeo & $\begin{array}{l}\text { Familias comparten sus experien- } \\
\text { cias para ver cómo poder seguir con } \\
\text { los aprendizajes en el día a día. } \\
\text { https://www.youtube.com/watch?v } \\
=\text { c5SRVzE3ITA\&feature=youtu.be } \\
\text { Recurso alineado con el paradig- } \\
\text { ma de intervención centrado en la } \\
\text { familia. }\end{array}$ \\
\hline 15 & $\begin{array}{c}4 \\
\text { For- } \\
\text { mación }\end{array}$ & $\begin{array}{l}20 / 3 / \\
2020\end{array}$ & $\begin{array}{c}\text { Plena } \\
\text { inclusión }\end{array}$ & Vídeo & $\begin{array}{l}\text { Conversación de Atención Tem- } \\
\text { prana frente al COVID-19: Estra- } \\
\text { tegias para acompañar a las familias } \\
\text { de manera online. } \\
\text { https://youtu.be/vbkA2GBC02o } \\
\text { Recurso alineado con el paradig- } \\
\text { ma de intervención centrado en la } \\
\text { familia. }\end{array}$ \\
\hline 16 & $\begin{array}{c}3 \\
\text { Re- } \\
\text { curs. } \\
\text { profes. }\end{array}$ & $\begin{array}{l}20 / 3 / \\
2020\end{array}$ & AEIPI & Doc & $\begin{array}{l}\text { Propuesta continuidad. } \\
\text { https://drive.google.com/file/ } \\
\text { d/1dHTc_Loi65zbqeCuNE6DE- } \\
\text { qDB1GSCNK1x/view } \\
\text { Recurso alineado con el paradig- } \\
\text { ma de intervención centrado en la } \\
\text { familia. }\end{array}$ \\
\hline 17 & $\begin{array}{c}2 \\
\text { Rec. } \\
\text { familia }\end{array}$ & $\begin{array}{l}21 / 3 / \\
2020\end{array}$ & UNICEF & Doc & $\begin{array}{l}\text { Guía COVID-19 para madres, pa- } \\
\text { dres y educadores. } \\
\text { https://www.unicef.es/publica- } \\
\text { cion/coronavirus-guia-para-ma- } \\
\text { dres-padres-y-educadores } \\
\text { Recurso que no se alinea con un pa- } \\
\text { radigma de intervención específico. }\end{array}$ \\
\hline
\end{tabular}




\begin{tabular}{|c|c|c|c|c|c|}
\hline \multicolumn{6}{|c|}{ TABLA 1. Descripción y enlaces de los recursos generados (cont.) } \\
\hline N. & $\begin{array}{c}\text { Temá- } \\
\text { tica }\end{array}$ & Fecha & Entidad & Tipo & Detalle y enlaces \\
\hline $\begin{array}{c}18 \\
a \\
25\end{array}$ & $\begin{array}{c}2 \\
\text { Rec. } \\
\text { familia }\end{array}$ & $\begin{array}{l}23 / 3 / \\
2020\end{array}$ & AVAPACE & Vídeo & $\begin{array}{l}\text { Se ponen en marcha “cafés online”, espe- } \\
\text { cialmente dirigidos a familias, de lunes a } \\
\text { jueves, para poder compartir entre ellas ex- } \\
\text { periencias, ideas e inquietudes. Se abordan } \\
\text { temas como el juego y la planificación del } \\
\text { tiempo, el control de esfínteres o descu- } \\
\text { briendo la comida. Los enlaces requieren } \\
\text { solicitar permiso para acceder al recurso: } \\
\text { 18. } 23 \text { de marzo: Juego y planificación del } \\
\text { tiempo en casa. https://drive.google. } \\
\text { com/a/avapace.org/file/d/1r3-Eci- } \\
\text { tkXQP6AvF82gAvQtMyr6RBzO5J/ } \\
\text { view?usp=drive_web } \\
\text { 19. 24 de marzo: Gestionar la ansiedad. } \\
\text { https://drive.google.com/a/avapace.org/ } \\
\text { file/d/1r3-EcitkXQP6AvF82gAvQtMy- } \\
\text { r6RBzO5J/view?usp=drive_web } \\
\text { 20. 25 de marzo: Estimulando la comuni- } \\
\text { cación de mi hijo/a a través del juego. } \\
\text { https://drive.google.com/open?id=1lguL } \\
\text { nHT6Iu3O8qGJTHJqOEjORByatYTk } \\
\text { 21. 26 de marzo: Descubriendo la comida. } \\
\text { https://drive.google.com/file/d/1s_vTv2S- } \\
\text { qXwWn5x7POaFvumtJVOzcH74j/view } \\
\text { 22. 30 de marzo: Ideas para el control de } \\
\text { esfínteres. https://drive.google.com/file/ } \\
\text { d/1BVg79pUQ0VZ5SH1FlstrxBQItcwi- } \\
\text { 74D/view?ts=5e82fof5 } \\
\text { 23. } 31 \text { de marzo: Taller mediado por familias } \\
\text { para el intercambio de estrategias. https:// } \\
\text { drive.google.com/a/avapace.org/file/d/13 } \\
\text { GXeO3x1gjKGcmyLjvkE88kw0xq3GW- } \\
\text { H/view?usp=drive_web } \\
\text { 24. } 7 \text { de abril: Estratégicas pedagógicas. } \\
\text { https://drive.google.com/a/avapace.org/ } \\
\text { file/d/1ebEtntIL-BYMXkXQzTGXF- } \\
\text { TyvN4krb66w/view?usp=drive_web } \\
\text { 25. } 8 \text { de abril: Vamos a dormir, calidad del } \\
\text { sueño. https://drive.google.com/a/avapace. } \\
\text { org/file/d/1JAH7byrh-5l9Cx4vYVZoD- } \\
\text { WKD-TRpSV1h/view?usp=drive_web } \\
\text { Recurso alineado con el paradigma de inter- } \\
\text { vención centrado en la familia. }\end{array}$ \\
\hline
\end{tabular}

Ediciones Universidad de Salamanca / CC BY-NC-ND

Siglo Cero, vol. 52, número extraordinario, 2021, pp. 37-74 


\begin{tabular}{|c|c|c|c|c|c|}
\hline \multicolumn{6}{|c|}{ TABLA 1. Descripción y enlaces de los recursos generados (cont.) } \\
\hline N. & $\begin{array}{l}\text { Temá- } \\
\text { tica }\end{array}$ & Fecha & Entidad & Tipo & Detalle y enlaces \\
\hline 26 & $\begin{array}{l}5 \text { Pla- } \\
\text { tafor- } \\
\text { mas }\end{array}$ & $\begin{array}{l}23 / 3 / \\
2020\end{array}$ & AEIPI & App & $\begin{array}{l}\text { AEIPI genera una aplicación a través } \\
\text { de la plataforma Twist para poder ge- } \\
\text { nerar un punto de encuentro y poder } \\
\text { compartir los recursos que se van ge- } \\
\text { nerando vía online. } \\
\text { https://drive.google.com/file/d/1Jvpr } \\
\text { _HFE0J99lvxP8GbCVOFQWzp-LW } \\
\text { H3/view?usp=sharing } \\
\text { Se comparte cómo realizar la interven- } \\
\text { ción a distancia, cómo realizar la coor- } \\
\text { dinación de equipo, qué canales pode- } \\
\text { mos establecer con la familia. } \\
\text { Recurso alineado con el paradigma de } \\
\text { intervención centrado en la familia. }\end{array}$ \\
\hline 27 & $\begin{array}{c}1 \\
\text { Situac. } \\
\text { centro }\end{array}$ & $\begin{array}{l}23 / 3 / \\
2020\end{array}$ & GAT & Doc & $\begin{array}{l}\text { Comunicado del GAT. [DECLARA- } \\
\text { CIÓN PANDEMIA COVID-19]. } \\
\text { http://gat-atenciontemprana.org/ } \\
\text { 2020/03/23/declaracion-pandemia- } \\
\text { covid-19/ } \\
\text { Recurso que no se alinea con un para- } \\
\text { digma de intervención específico. }\end{array}$ \\
\hline 28 & $\begin{array}{c}1 \\
\text { Situac. } \\
\text { centro }\end{array}$ & $\begin{array}{l}23 / 3 / \\
2020\end{array}$ & AEIPI & App & $\begin{array}{l}\text { AEIPI lanza una encuesta para conocer } \\
\text { la situación global de los centros, la en- } \\
\text { cuesta en } 6 \text { días es contestada por } 104 \\
\text { personas, donde un } 89 \% \text { estaba reali- } \\
\text { zando teletrabajo y solamente } 5 \text { perso- } \\
\text { nas no tenían contacto con las familias. } \\
\text { Recurso que no se alinea con un para- } \\
\text { digma de intervención específico. }\end{array}$ \\
\hline 29 & $\begin{array}{c}3 \\
\text { Re- } \\
\text { curs. } \\
\text { profes. }\end{array}$ & $\begin{array}{l}23 / 3 / \\
2020\end{array}$ & $\begin{array}{c}\text { Robin } \\
\text { McWilliam }\end{array}$ & Doc & $\begin{array}{l}\text { Autores específicos de modelos de in- } \\
\text { tervención aportan su visión y adapta- } \\
\text { ción a esta nueva situación: Teleinter- } \\
\text { vención y el modelo basado en rutinas. } \\
\text { https://naturalenvironments.blogspot. } \\
\text { com/2020/03/tele-intervention-and- } \\
\text { routines-based.html?spref=fb\&m }=1 \\
\text { Recurso alineado con el paradigma de } \\
\text { intervención centrado en la familia. }\end{array}$ \\
\hline
\end{tabular}




\begin{tabular}{|c|c|c|c|c|c|}
\hline \multicolumn{6}{|c|}{ TABLA 1. Descripción y enlaces de los recursos generados (cont.) } \\
\hline N. & $\begin{array}{l}\text { Temá- } \\
\text { tica }\end{array}$ & Fecha & Entidad & Tipo & Detalle y enlaces \\
\hline 30 & $\begin{array}{c}3 \\
\text { Recurs. } \\
\text { profes. }\end{array}$ & $\begin{array}{l}23 / 3 / \\
2020\end{array}$ & $\begin{array}{l}\text { ECTA } \\
\text { Center }\end{array}$ & Doc & $\begin{array}{l}\text { Planificación para el uso de video- } \\
\text { conferencias para visitas domicilia- } \\
\text { rias de intervención temprana du- } \\
\text { rante la pandemia COVID-19. } \\
\text { https://ectacenter.org/ pdfs/to- } \\
\text { pics/disaster/Planning_for_the_- } \\
\text { Use_of_Video_Conferencing_in_- } \\
\text { EI_during_COVID-19_Pandemic. } \\
\text { pdf } \\
\text { Recurso alineado con el paradigma de } \\
\text { intervención centrado en la familia. }\end{array}$ \\
\hline 31 & $\begin{array}{c}2 \\
\text { Rec. } \\
\text { familia }\end{array}$ & $\begin{array}{l}23 / 3 / \\
2020\end{array}$ & $\begin{array}{c}\text { EOEP } \\
\text { Específico } \\
\text { Murcia }\end{array}$ & Doc & $\begin{array}{l}\text { Listado de APPs por categorías. } \\
\text { https://drive.google.com/open?id= } \\
\text { 1EOULDtFCYZNmAfdw4DWAc } \\
\text { LrW_FvEZ7lo } \\
\text { EOEP Específico de Autismo y } \\
\text { otros Trastornos Graves del Desa- } \\
\text { rrollo de Murcia. } \\
\text { Recurso que no se alinea con un pa- } \\
\text { radigma de intervención específico. }\end{array}$ \\
\hline 32 & $\begin{array}{c}2 \\
\text { Rec. } \\
\text { familia }\end{array}$ & $\begin{array}{l}23 / 3 / \\
2020\end{array}$ & UNICEF & Doc & $\begin{array}{l}\text { Educación contra el coronavirus: } 10 \\
\text { tareas de la rutina diaria. } \\
\text { https://www. unicef.es/educa/ } \\
\text { blog/10-tareas-rutina-diaria-familia } \\
\text { Recurso que no se alinea con un pa- } \\
\text { radigma de intervención específico. }\end{array}$ \\
\hline 33 & $\begin{array}{c}1 \\
\text { Situac. } \\
\text { centro }\end{array}$ & $\begin{array}{l}23 / 3 / \\
2020\end{array}$ & DMCN & Vídeo & $\begin{array}{l}\text { Derechos Humanos de los Niños } \\
\text { con Discapacidad: el Desafío del } \\
\text { COVID-19|Verónica Schiariti. } \\
\text { https://www.youtube.com/watch?v } \\
\text { =qIOr9xSB7RQ\&feature=youtu.be } \\
\text { Recurso que no se alinea con un pa- } \\
\text { radigma de intervención específico. }\end{array}$ \\
\hline 34 & $\begin{array}{c}4 \\
\text { For- } \\
\text { mación }\end{array}$ & $\begin{array}{l}24 / 3 / \\
2020\end{array}$ & $\begin{array}{l}\text { Plena inclu- } \\
\text { sión }\end{array}$ & Vídeo & $\begin{array}{l}\text { Seminario online "Estar bien en } \\
\text { casa". } \\
\text { https://youtu.be/DIQ3HuE6kGI } \\
\text { Recurso que no se alinea con un pa- } \\
\text { radigma de intervención específico. }\end{array}$ \\
\hline
\end{tabular}




\begin{tabular}{|c|c|c|c|c|c|}
\hline \multicolumn{6}{|c|}{ TABLA 1. Descripción y enlaces de los recursos generados (cont.) } \\
\hline N. & $\begin{array}{l}\text { Temá- } \\
\text { tica }\end{array}$ & Fecha & Entidad & Tipo & Detalle y enlaces \\
\hline 35 & $\begin{array}{c}2 \\
\text { Rec. } \\
\text { familia }\end{array}$ & $\begin{array}{l}24 / 3 / \\
2020\end{array}$ & $?$ & Doc & $\begin{array}{l}\text { Consejos para cuidadores de niños y } \\
\text { niñas durante confinamiento. } \\
\text { https://drive.google.com/file/ } \\
\text { d/1JSOBbBSI9j92hm4_McFt_ } \\
\text { WcUlY9286uQ/view?usp=sharing } \\
\text { Recurso que no se alinea con un pa- } \\
\text { radigma de intervención específico. }\end{array}$ \\
\hline 36 & $\begin{array}{c}2 \\
\text { Rec. } \\
\text { familia }\end{array}$ & $\begin{array}{l}24 / 3 / \\
2020\end{array}$ & *Equipo AT & Doc & $\begin{array}{l}\text { Actividades para casa con bebés, ni- } \\
\text { ños 1-2 y 2-3 años. } \\
\text { https://drive.google.com/drive/ } \\
\text { folders/155PUyfBaexZf9wDetr6q- } \\
\text { tn473Tohj7J?usp=sharing } \\
\text { *Equipo AT San Sebastián de los } \\
\text { Reyes-Alcobendas (Madrid). } \\
\text { Recurso que no se alinea con un pa- } \\
\text { radigma de intervención específico. }\end{array}$ \\
\hline 37 & $\begin{array}{c}2 \\
\text { Rec. } \\
\text { familia }\end{array}$ & $\begin{array}{l}24 / 3 / \\
2020\end{array}$ & $\begin{array}{l}\text { @Mother- } \\
\text { could }\end{array}$ & Doc & $\begin{array}{l}\text { Recetas sensoriales. } \\
\text { h t t p s : / / d rive.g o o g l e.c o m / } \\
\text { open?id=13-sQbpd-2-ha1 vowX3I- } \\
\text { VWecBxKcYc57- } \\
\text { Recurso que no se alinea con un pa- } \\
\text { radigma de intervención específico. }\end{array}$ \\
\hline 38 & $\begin{array}{c}2 \\
\text { Rec. } \\
\text { profes. }\end{array}$ & $\begin{array}{l}24 / 3 / \\
2020\end{array}$ & $\begin{array}{l}\text { Zero to } \\
\text { Three }\end{array}$ & App & $\begin{array}{l}\text { Recursos sobre coronavirus para } \\
\text { profesionales de la primera infancia. } \\
\text { https://www.z erotothree.org/ } \\
\text { resources/3291-coronavirus-resour- } \\
\text { ces-for-early-childhood-professionals } \\
\text { Recurso alineado con el paradigma de } \\
\text { intervención centrado en la familia. }\end{array}$ \\
\hline 39 & $\begin{array}{c}4 \\
\text { For- } \\
\text { mación }\end{array}$ & $\begin{array}{l}25 / 3 / \\
2020\end{array}$ & AEIPI & Vídeo & $\begin{array}{l}\text { Varios profesionales expertos en el } \\
\text { campo de la Atención Temprana, de } \\
\text { manera desinteresada, comienzan a } \\
\text { compartir consejos para orientar tan- } \\
\text { to a profesionales como a familias, } \\
\text { como el ejemplo de Pau García y Ca- } \\
\text { talina Morales, con el tema: “Atención } \\
\text { Temprana a Distancia es Posible”. } \\
\text { https://vimeo.com/400358242 } \\
\text { Recurso alineado con el paradigma de } \\
\text { intervención centrado en la familia. }\end{array}$ \\
\hline
\end{tabular}




\begin{tabular}{|c|c|c|c|c|c|}
\hline N. & $\begin{array}{c}\text { Temá- } \\
\text { tica }\end{array}$ & Fecha & Entidad & Tipo & Detalle y enlaces \\
\hline 40 & $\begin{array}{c}2 \\
\text { Rec. } \\
\text { familia }\end{array}$ & $\begin{array}{l}25 / 3 / \\
2020\end{array}$ & *Asociación & Doc & $\begin{array}{l}\text { Programa de Ocio en Cuarentena. } \\
\text { https://drive.google.com/file/ } \\
\text { d/1blJVwsmFQzTmYAooE6shY01PC } \\
\text { zFmM-qR/view?usp=sharing } \\
\text { *Asociación Asperger Murcia y FAUM. } \\
\text { Recurso que no se alinea con un paradig- } \\
\text { ma de intervención específico. }\end{array}$ \\
\hline 41 & $\begin{array}{c}2 \\
\text { Rec. } \\
\text { familia }\end{array}$ & $\begin{array}{l}25 / 3 / \\
2020\end{array}$ & $\begin{array}{c}\text { Equipo } \\
\text { Patios } \\
\text { y Parques } \\
\text { Dinámicos }\end{array}$ & App & $\begin{array}{l}\text { Recurso totalmente gratuito que nace de } \\
\text { la necesidad de convertir estos días de en- } \\
\text { cierro, incertidumbre y agobio en días de } \\
\text { diversión y juego en familia. } \\
\text { http://yojuegoencasa.es/ } \\
\text { Recurso que no se alinea con un paradig- } \\
\text { ma de intervención específico. }\end{array}$ \\
\hline 42 & $\begin{array}{c}1 \\
\text { Situac. } \\
\text { centros }\end{array}$ & $\begin{array}{l}26 / 3 / \\
2020\end{array}$ & GEN-CAT & Doc & $\begin{array}{l}\text { Cataluña asegura el pago a las entidades y } \\
\text { a los CDIAT. Treball, Afers Socials i Fa- } \\
\text { mílies garanteix el pagament a les entitats } \\
\text { proveiddores de serveis socials i activa tota } \\
\text { la xarxa assistencial. } \\
\text { https://govern.cat/salapremsa/notes- } \\
\text { premsa/383626/treball-afers-socials- } \\
\text { families-garanteix-pagament-entitats- } \\
\text { proveidores-serveis-socials-activa-tota- } \\
\text { xarxa-assistencial } \\
\text { Recurso que no se alinea con un paradig- } \\
\text { ma de intervención específico. }\end{array}$ \\
\hline
\end{tabular}




\begin{tabular}{|c|c|c|c|c|c|}
\hline N. & Temática & Fecha & Entidad & Tipo & Detalle y enlaces \\
\hline $\begin{array}{c}43 \\
\mathrm{a} \\
48\end{array}$ & $\begin{array}{c}4 \\
\text { For } \\
\text { mación }\end{array}$ & $\begin{array}{l}27 / 3 / \\
2020\end{array}$ & $\begin{array}{c}\text { Diferentes } \\
\text { grupos }\end{array}$ & Vídeo & $\begin{array}{l}\text { Desde esta fecha se van sucediendo } \\
\text { numerosas charlas con un formato } \\
\text { abierto con carácter gratuito de los } \\
\text { diferentes perfiles profesionales que } \\
\text { conforman la atención temprana. } \\
\text { 43. } 27 \text { de marzo: Teleton Paraguay: } \\
\text { Explicación de las Prácticas } \\
\text { Centradas en la Familia - Marisu } \\
\text { Pedernera. https://www.facebook. } \\
\text { com/TeletonParaguay/videos/257 } \\
\text { 6650629290611/?vh=e\&d=n } \\
\text { Recurso alineado con el paradig- } \\
\text { ma de intervención centrado en } \\
\text { la familia. } \\
\text { 44. 27 de marzo: Dolor Infantil } \\
\text { - https://youtu.be/-2G9SbL- } \\
\text { HuUE - Guillermo Ceniza-Bor- } \\
\text { dallo, Coord.: Álvaro Hidalgo. } \\
\text { 45. } 7 \text { de abril: Escoliosis - Guía } \\
\text { SOSORT https://youtu.be/ } \\
\text { DYQJoutYvnM -Ana León, } \\
\text { Coord.: Álvaro Hidalgo. } \\
\text { 46. 10 de abril: Herramientas para } \\
\text { favorecer el desarrollo motriz } \\
\text { en el primer año de vida - Rocio } \\
\text { Palomo Carrión. } \\
\text { - Parte 1: https://youtu.be/ajDRZ- } \\
\text { nf4wcQ } \\
\text { - Parte 2: https://youtu.be/CqFxs- } \\
\text { q16yPc } \\
\text { - Parte 3: https://youtu.be/ } \\
\text { od2XAIr-ms4 } \\
\text { 47. 17 de abril: Implementación de } \\
\text { detección temprana en niños de alto } \\
\text { riesgo neurológico. https://youtu. } \\
\text { be/_iub OBoXumI - Álvaro Hidal- } \\
\text { go y Javier Merino, Coord.: Paqui } \\
\text { Cañete. } \\
\text { Recursos que no se alinean con un pa- } \\
\text { radigma de intervención específico. } \\
\end{array}$ \\
\hline
\end{tabular}




\begin{tabular}{|c|c|c|c|c|c|}
\hline N. & $\begin{array}{l}\text { Temá- } \\
\text { tica }\end{array}$ & Fecha & Entidad & Tipo & Detalle y enlaces \\
\hline 49 & $\begin{array}{c}2 \\
\text { Rec. } \\
\text { familia }\end{array}$ & $\begin{array}{l}27 / 3 / \\
2020\end{array}$ & $\begin{array}{l}\text { Path- } \\
\text { ways }\end{array}$ & App & $\begin{array}{l}\text { AEIPI comparte enlace de recomendacio- } \\
\text { nes para fomentar el movimiento en los } \\
\text { niños. } \\
\text { https://pathways.org/ } \\
\text { Pathways.org se plantea como misión pro- } \\
\text { porcionar herramientas libres para maxi- } \\
\text { mizar el desarrollo motor, sensorial y de la } \\
\text { comunicación de todos los niños. Especifi- } \\
\text { ca que su visión es empoderar a los padres } \\
\text { para comprender y que estimulen el desa- } \\
\text { rrollo de sus bebés para mantenerlos enca- } \\
\text { minados o detectar precozmente posibles } \\
\text { retrasos. } \\
\text { Recurso alineado con el paradigma de in- } \\
\text { tervención centrado en la familia. }\end{array}$ \\
\hline 50 & $\begin{array}{c}2 \\
\text { Rec. } \\
\text { familia }\end{array}$ & $29 / 3 / 20$ & $\begin{array}{l}\text { ECTA } \\
\text { Center }\end{array}$ & App & $\begin{array}{l}\text { AEIPI comparte las guías familiares de } \\
\text { ECTA Center. } \\
\text { https://ectacenter.org/decrp/type-pgfa- } \\
\text { mily-sp.asp } \\
\text { Recurso alineado con el paradigma de in- } \\
\text { tervención centrado en la familia. }\end{array}$ \\
\hline 51 & $\begin{array}{c}1 \\
\text { Situac. } \\
\text { centros }\end{array}$ & $\begin{array}{l}30 / 3 / \\
2020\end{array}$ & AEIPI & $\begin{array}{l}\text { Vídeo } \\
\text { X } 4\end{array}$ & $\begin{array}{l}\text { Experiencias de Centros de Atención } \\
\text { Temprana por el COVID-19. } \\
\text { Se publica la situación de cuatros centros } \\
\text { de Atención Temprana y cómo se han } \\
\text { adaptado a la nueva situación (ASPACE } \\
\text { Guipúzcoa; Equipo InSitu Crevillente - } \\
\text { Alicante: CDIAT Tarancón - Castilla-La } \\
\text { Mancha; ADISLAN - Lanzarote). } \\
\text { Equipo InSitu - Alicante - https://youtu. } \\
\text { be/3WbI_QZDw38 - 5 de marzo } \\
\text { CDIAT Tarancón - Castilla-La Mancha } \\
\text { - https://youtu.be/eQGatCtCzao - 22 de } \\
\text { marzo } \\
\text { ADISLAN - Lanzarote - https://youtu. } \\
\text { be/EvnLHN3XUDg - 23 de marzo } \\
\text { Aspace Guipuzkoa - https://youtu.be/ } \\
\text { MGtfNz7EWnA - 27 de marzo } \\
\text { Recurso alineado con el paradigma de } \\
\text { intervención centrado en la familia. }\end{array}$ \\
\hline
\end{tabular}




\begin{tabular}{|c|c|c|c|c|c|}
\hline \multicolumn{6}{|c|}{ TABLA 1. Descripción y enlaces de los recursos generados (cont.) } \\
\hline N. & $\begin{array}{l}\text { Temá- } \\
\text { tica }\end{array}$ & Fecha & Entidad & Tipo & Detalle y enlaces \\
\hline 52 & $\begin{array}{c}3 \\
\text { Rec. } \\
\text { profes. }\end{array}$ & $\begin{array}{l}30 / 3 / \\
2020\end{array}$ & $\begin{array}{c}\text { Plena } \\
\text { inclusión }\end{array}$ & Doc & $\begin{array}{l}\text { Guía de Apoyos en casa durante el co- } \\
\text { ronavirus. } \\
\text { https://www.plenainclusion.org/sites/ } \\
\text { default/files/guia_apoyos_en_casa_du- } \\
\text { rante_el_coronavirus.pdf } \\
\text { Recurso que no se alinea con un paradig- } \\
\text { ma de intervención específico. }\end{array}$ \\
\hline 53 & $\begin{array}{c}2 \\
\text { Rec. } \\
\text { familia }\end{array}$ & $\begin{array}{c}30 / 3 / \\
20\end{array}$ & TRASTEA & Doc & $\begin{array}{l}\text { TRASTEA. Niños con TEA durante el } \\
\text { confinamiento } \\
\text { https://drive.google.com/file/d/1X } \\
\text { HRwEl8foHqZqL71l-MLyqrBQfdb- } \\
\text { CSwv/view ?usp=sharing } \\
\text { Recurso que no se alinea con un paradig- } \\
\text { ma de intervención específico. }\end{array}$ \\
\hline 54 & $\begin{array}{c}4 \\
\text { Forma- } \\
\text { ción }\end{array}$ & $\begin{array}{l}31 / 3 / \\
2020\end{array}$ & $\begin{array}{l}\text { ACAIT } \\
\text { Málaga } \\
\text { y Equipo } \\
\text { SIDI }\end{array}$ & Vídeo & $\begin{array}{l}\text { Apoyamos a las familias con la teleinter- } \\
\text { vención- } \\
\text { https://www.youtube.com/watch?v=12 } \\
\text { uyhoJLAQk\&feature=youtu.be } \\
\text { Recurso alineado con el paradigma de } \\
\text { intervención centrado en la familia. }\end{array}$ \\
\hline 55 & $\begin{array}{c}2 \\
\text { Rec. } \\
\text { familia }\end{array}$ & $\begin{array}{l}31 / 3 / \\
2020\end{array}$ & $\begin{array}{l}\text { Equipo } \\
\text { InSitu }\end{array}$ & Doc & $\begin{array}{l}\text { Comienzan a lanzar infografías para re- } \\
\text { flejar la cantidad de aprendizajes que se } \\
\text { pueden dar en la vida cotidiana. } \\
\text { https://drive.google.com/drive/folders } \\
\text { /1--n6uOeUGVaiiJzSKYCkYm_Fbtet8 } \\
864 \text { ?usp=sharing } \\
\text { Recurso alineado con el paradigma de } \\
\text { intervención centrado en la familia. }\end{array}$ \\
\hline 56 & $\begin{array}{c}4 \\
\text { Forma- } \\
\text { ción }\end{array}$ & $\begin{array}{l}1 / 4 / \\
2020\end{array}$ & $\begin{array}{c}\text { Plena } \\
\text { inclusión }\end{array}$ & Vídeo & $\begin{array}{l}\text { Tele Intervención en Atención Tempra- } \\
\text { na. Sesión } 1 . \\
\text { https://youtu.be/TmCf_NNuY_w } \\
\text { Recurso alineado con el paradigma de } \\
\text { intervención centrado en la familia. }\end{array}$ \\
\hline 57 & $\begin{array}{c}4 \\
\text { Forma- } \\
\text { ción }\end{array}$ & $\begin{array}{l}2 / 4 / \\
2020\end{array}$ & $\begin{array}{l}\text { ACAIT } \\
\text { Málaga y } \\
\text { Equipo } \\
\text { SIDI }\end{array}$ & Vídeo & $\begin{array}{l}\text { ¿Cómo realizar la tele práctica en AT a } \\
\text { distancia? } \\
\text { https://youtu.be/60vIgLCiVAA } \\
\text { Recurso alineado con el paradigma de } \\
\text { intervención centrado en la familia. }\end{array}$ \\
\hline
\end{tabular}




\begin{tabular}{|c|c|c|c|c|c|}
\hline \multicolumn{6}{|c|}{ TABLA 1. Descripción y enlaces de los recursos generados (cont.) } \\
\hline N. ${ }^{\circ}$ & $\begin{array}{c}\text { Temá- } \\
\text { tica }\end{array}$ & Fecha & Entidad & Tipo & Detalle y enlaces \\
\hline 58 & $\begin{array}{c}2 \\
\text { Rec. } \\
\text { familia }\end{array}$ & $\begin{array}{l}2 / 4 / \\
2020\end{array}$ & UFV & Doc & $\begin{array}{l}\text { Películas para ver en familia } \\
\text { https://drive.google.com/file/d/1 arY } \\
\text { ucVH-cbHXgq5TDcpkI5h_tbigepN_/vi } \\
\text { ew?usp=sharing } \\
\text { Recurso que no se alinea con un paradigma } \\
\text { de intervención específico. }\end{array}$ \\
\hline 59 & $\begin{array}{c}4 \\
\text { Forma- } \\
\text { ción }\end{array}$ & $\begin{array}{l}2 / 4 / \\
2020\end{array}$ & $\begin{array}{l}\text { Down } \\
\text { España }\end{array}$ & $\begin{array}{c}\text { Doc } \\
\text { Vídeo }\end{array}$ & $\begin{array}{l}\text { Juego en familia. } \\
\text { https://www.sindromedown.net/noticia/ } \\
\text { sonsoles-perpinan-jugar-es-algo-absoluta- } \\
\text { mente-necesario-que-prepara-a-los-ninos- } \\
\text { para-su-futuro/ } \\
\text { Recurso que no se alinea con un paradigma } \\
\text { de intervención específico. }\end{array}$ \\
\hline 60 & $\begin{array}{c}2 \\
\text { Rec. } \\
\text { familia } \\
3 \text { Rec. } \\
\text { profes. }\end{array}$ & $\begin{array}{l}3 / 4 / \\
2020\end{array}$ & $\begin{array}{l}\text { Illinois } \\
\text { Early } \\
\text { Inter- } \\
\text { vention } \\
\text { Clea- } \\
\text { ringhou- } \\
\text { se } \\
\end{array}$ & App & $\begin{array}{l}\text { AEIPI Comienza la difusión de infografías } \\
\text { de Illinois Early Intervention Clearinghou- } \\
\text { se, basadas en el potencial de las actividades } \\
\text { cotidianas. } \\
\text { https://eiclearinghouse.org/es/ } \\
\text { Recurso alineado con el paradigma de in- } \\
\text { tervención centrado en la familia. }\end{array}$ \\
\hline 61 & $\begin{array}{c}2 \\
\text { Rec. } \\
\text { familia }\end{array}$ & $\begin{array}{l}6 / 4 / \\
2020\end{array}$ & AEPap* & Doc & $\begin{array}{l}\text { La AEPap, en su sección de Familia y Sa- } \\
\text { lud de su página web, publica un artículo } \\
\text { titulado "Atención Temprana versus Coro- } \\
\text { navirus" invitando a las familias a seguir en } \\
\text { contacto, por medios telemáticos, con sus } \\
\text { terapeutas de Atención Temprana. } \\
\text { https://www.familiaysalud.es/sintomas- } \\
\text { y-enfermedades/infecciones/todo-sobre- } \\
\text { el-coronavirus/atencion-temprana-versus- } \\
\text { coronavirus } \\
\text { *Asociación Española de Pediatría de Aten- } \\
\text { ción Primaria. } \\
\text { Recurso que no se alinea con un paradigma } \\
\text { de intervención específico. }\end{array}$ \\
\hline 62 & $\begin{array}{c}1 \\
\text { Situac. } \\
\text { centros }\end{array}$ & $\begin{array}{l}7 / 4 / \\
2020\end{array}$ & $\begin{array}{c}\text { ACAIT } \\
\text { Málaga y } \\
\text { Equipo } \\
\text { SIDI }\end{array}$ & Doc & $\begin{array}{l}\text { Protocolos de Atención Temprana duran- } \\
\text { te el aislamiento por COVID-19. ACAIT } \\
\text { Málaga y Equipo SIDI. } \\
\text { https://drive.google.com/file/d/19IeF_uY } \\
\text { HJBRbo531yLOLXfLpLaZkiu-P/vi } \\
\text { ew?usp=sharing } \\
\text { Recurso alineado con el paradigma de in- } \\
\text { tervención centrado en la familia. }\end{array}$ \\
\hline
\end{tabular}




\begin{tabular}{|c|c|c|c|c|c|}
\hline \multicolumn{6}{|c|}{ TABLA 1. Descripción y enlaces de los recursos generados (cont.) } \\
\hline N. ${ }^{\circ}$ & $\begin{array}{c}\text { Temá- } \\
\text { tica }\end{array}$ & Fecha & Entidad & Tipo & Detalle y enlaces \\
\hline 63 & $\begin{array}{l}1 \\
\text { Situac. } \\
\text { centros } \\
\quad 4 \\
\text { Recurs. } \\
\text { profes. }\end{array}$ & $\begin{array}{l}7 / 4 / \\
2020\end{array}$ & AGAT* & Doc & $\begin{array}{l}\text { Atención temprana en tiempo de confina- } \\
\text { miento. } \\
\text { http://atenciontemprana.com/atencion-tem- } \\
\text { prana-en-tiempo-de-confinamiento/ } \\
\text { "Asociación Gallega de Atención Temprana. } \\
\text { Recurso alineado con el paradigma de inter- } \\
\text { vención ambulatoria y centrada en el niño. }\end{array}$ \\
\hline 64 & $\begin{array}{c}4 \\
\text { For- } \\
\text { mación }\end{array}$ & $\begin{array}{l}8 / 4 / \\
2020\end{array}$ & $\begin{array}{c}\text { Plena } \\
\text { inclusión }\end{array}$ & Vídeo & $\begin{array}{l}\text { Tele Intervención en Atención Temprana. } \\
\text { Sesión } 2 \text {. } \\
\text { https://youtu.be/7bkq2LhXcoE } \\
\text { Recurso alineado con el paradigma de in- } \\
\text { tervención centrado en la familia. }\end{array}$ \\
\hline 65 & $\begin{array}{c}3 \\
\text { Recurs. } \\
\text { profes. }\end{array}$ & $\begin{array}{l}13 / 4 / \\
2020\end{array}$ & AEIPI & App & $\begin{array}{l}\text { AEIPI lanza la campaña de felicitar a los } \\
\text { profesionales de Atención Temprana por } \\
\text { su trabajo compaginando el apoyo a dis- } \\
\text { tancia a las familias y estar compaginando } \\
\text { con su vida personal. } \\
\text { https://twitter.com/AEIPI_ORG/sta- } \\
\text { tus/1249584126732222464 } \\
\text { Recurso que no se alinea con un paradigma } \\
\text { de intervención específico. }\end{array}$ \\
\hline 66 & $\begin{array}{c}1 \\
\text { Situac. } \\
\text { centros }\end{array}$ & $\begin{array}{l}13 / 4 / \\
2020\end{array}$ & Heraldo & Doc & $\begin{array}{l}\text { El cierre de colegios y centros de rehabili- } \\
\text { tación pone en riesgo las terapias de niños } \\
\text { con enfermedades raras. } \\
\text { https://www.heraldo.es/noticias/salud/ } \\
\text { 2020/04/13/el-cierre-de-colegios-pone-en- } \\
\text { riesgo-las-terapias-de-ninos-con-enferme } \\
\text { dades-raras-1369335.html?utm_source= } \\
\text { twitter.com \&utm_medium=social } \\
\text { share\&utm_campaign=mobile_web } \\
\text { Recurso que no se alinea con un paradigma } \\
\text { de intervención específico. }\end{array}$ \\
\hline 67 & $\begin{array}{c}4 \\
\text { For- } \\
\text { mación }\end{array}$ & $\begin{array}{l}14 / 4 / \\
2020\end{array}$ & $\begin{array}{l}\text { ACAIT } \\
\text { Málaga, } \\
\text { Equipo } \\
\text { SIDI y } \\
\text { AEIPI }\end{array}$ & Vídeo & $\begin{array}{l}\text { Se inician Encuentros de Profesionales de } \\
\text { Atención Temprana diarios de } 45 \text { minutos. } \\
\text { https:// w w w.instagram.com/p/B - } \\
\text { 7IHEMJOA7/?igshid=1x3pkhz9dbp6i } \\
\text { Recurso alineado con el paradigma de in- } \\
\text { tervención centrado en la familia. }\end{array}$ \\
\hline
\end{tabular}




\begin{tabular}{|c|c|c|c|c|c|}
\hline N. ${ }^{\circ}$ & $\begin{array}{c}\text { Temá- } \\
\text { tica }\end{array}$ & Fecha & Entidad & Tipo & Detalle y enlaces \\
\hline 68 & $\begin{array}{c}4 \\
\text { For- } \\
\text { mación }\end{array}$ & $\begin{array}{l}15 / 4 / \\
2020\end{array}$ & $\begin{array}{c}\text { Plena } \\
\text { inclusión }\end{array}$ & Vídeo & $\begin{array}{l}\text { Tele Intervención en Atención Tempra- } \\
\text { na. Sesión } 3 \text {. } \\
\text { https://youtu.be/f7gyKmKBUV8 } \\
\text { Recurso alineado con el paradigma de } \\
\text { intervención centrado en la familia. }\end{array}$ \\
\hline 69 & $\begin{array}{c}2 \\
\text { Rec. } \\
\text { familia }\end{array}$ & $\begin{array}{l}15 / 4 / \\
2020\end{array}$ & $\begin{array}{l}\text { Ed. Inf.y } \\
\text { Aplica- } \\
\text { ciones } \\
\text { Web }\end{array}$ & App & $\begin{array}{l}\text { \#YoJuegoEnCasa. } \\
\text { Ciclo Superior en Educación Infantil, en } \\
\text { colaboración con el Ciclo de Desarrollo } \\
\text { de Aplicaciones Web. } \\
\text { https://sites.google.com/eusa.es/yojue- } \\
\text { goencasa/inicio } \\
\text { Recurso que no se alinea con un paradig- } \\
\text { ma de intervención específico. }\end{array}$ \\
\hline 70 & $\begin{array}{c}3 \\
\text { Re- } \\
\text { curs. } \\
\text { profes. }\end{array}$ & $\begin{array}{l}16 / 4 / \\
2020\end{array}$ & $\begin{array}{l}\text { Grupo } \\
\text { AMAS }\end{array}$ & Doc & $\begin{array}{l}\text { LOPD - Protocolos de confidencialidad } \\
\text { de datos. } \\
\text { https://drive.google.com/drive/folders/ } \\
\text { 1yr49iu5OLDbplK1TLskwWvzG5wm } \\
\text { cZtDD?usp=sharing } \\
\text { Recurso que no se alinea con un paradig- } \\
\text { ma de intervención específico. }\end{array}$ \\
\hline 71 & $\begin{array}{c}3 \\
\text { Re- } \\
\text { curs. } \\
\text { profes. }\end{array}$ & $\begin{array}{l}16 / 4 / \\
2020\end{array}$ & $\begin{array}{l}\text { Equipo } \\
\text { Recrea }\end{array}$ & Doc & $\begin{array}{l}\text { Propuesta Entrevista Inicial. Nuevas fa- } \\
\text { milias. Periodo de confinamiento. } \\
\text { https://drive.google.com/drive/folders/ } \\
\text { 16s2yI4FyQZzXr9rPjK22KNOFrVuE } \\
\text { HoLC?usp=sharing } \\
\text { Recurso alineado con el paradigma de } \\
\text { intervención centrado en la familia. }\end{array}$ \\
\hline 72 & $\begin{array}{c}1 \\
\text { Situac. } \\
\text { centro }\end{array}$ & $\begin{array}{l}17 / 4 / \\
2020\end{array}$ & AEIPI & Doc & $\begin{array}{l}\text { AEIPI difunde el Manifiesto de Aten- } \\
\text { ción Temprana. } \\
\text { https://bit.ly/manifiesto-aeipi } \\
\text { Recurso alineado con el paradigma de } \\
\text { intervención centrado en la familia. }\end{array}$ \\
\hline
\end{tabular}

Fuente: Elaboración propia. 
Los 72 recursos se reparten en un total de 33 documentos de texto (45,2\%), un número similar de 32 videoconferencias o seminarios online $(43,8 \%)$ y 8 aplicaciones o recursos web $(10,9 \%)$. Es de señalar que todos ellos, incluidos las videoconferencias y seminarios online, fueron siempre de acceso gratuito. En la Tabla 2 se recoge la distribución de los diferentes tipos de recursos en cada una de las 5 semanas analizadas. Se observa que el mayor volumen se generó en la segunda y tercera semana tras el confinamiento $(61,6 \%$ de los ellos se publicaron en esas dos semanas).

\begin{tabular}{|c|c|c|c|c|c|c|c|}
\hline \multicolumn{7}{|c|}{$\begin{array}{l}\text { Tabla 2. Distribución de los recursos (frecuencia y porcentaje) } \\
\text { en cada semana según el tipo de material aportado }\end{array}$} \\
\hline $\begin{array}{c}\text { Tipo de material } \\
\text { aportado }\end{array}$ & & $\begin{array}{c}\text { Semana } \\
1\end{array}$ & $\begin{array}{c}\text { Semana } \\
2\end{array}$ & $\begin{array}{c}\text { Semana } \\
3\end{array}$ & $\begin{array}{c}\text { Semana } \\
4\end{array}$ & $\begin{array}{c}\text { Semana } \\
5\end{array}$ & Total \\
\hline \multirow{2}{*}{ Documento escrito } & $\mathrm{n}$ & 7 & 12 & $7 *$ & 3 & 4 & 33 \\
& $\%$ & $9,6 \%$ & $16,4 \%$ & $9,6 \%$ & $4,1 \%$ & $5,5 \%$ & $45,2 \%$ \\
\hline Videoconferencia & $\mathrm{n}$ & 2 & 11 & $10 * \#$ & 5 & 4 & 32 \\
\hline \multirow{2}{*}{ Aplicación o recurso web } & $\mathrm{n}$ & $2,7 \%$ & $15,1 \%$ & $13,7 \%$ & $6,8 \%$ & $5,5 \%$ & $43,8 \%$ \\
\hline \multirow{2}{*}{ TOTAL } & $\mathrm{n}$ & $1,4 \%$ & $4,1 \%$ & $2,7 \%$ & 0 & 2 & 8 \\
\hline
\end{tabular}

*Un recurso es a la vez documento y videoconferencia.

\# Un recurso incluye cuatro vídeos grabados en diferentes fechas. No obstante, se contabiliza como un solo recurso ya que se facilitó el acceso a todos ellos en la misma fecha.

Fuente: Elaboración propia. 
En la Tabla 3 se recoge la distribución de los recursos en cada semana atendiendo a la orientación de su contenido. Se observa que aquellos relativos a la situación de los centros se concentran en las dos primeras semanas. En ellas encontramos 10 de los 15 recursos contabilizados de este tipo (lo que supone el 66,7 \%). También en ellas se concentra el $100 \%$ de las plataformas creadas. Por su parte, los recursos orientados a las familias, a los profesionales y a su formación surgen fundamentalmente a partir de la segunda semana.

\begin{tabular}{|c|c|c|c|c|c|c|}
\hline \multicolumn{7}{|c|}{$\begin{array}{l}\text { TABLA 3. Distribución (frecuencia y porcentajes) de los recursos } \\
\text { en cada semana según su contenido principal }\end{array}$} \\
\hline $\begin{array}{l}\text { Contenido principal } \\
\text { del recurso }\end{array}$ & $\begin{array}{c}\text { Semana } \\
1 \\
\end{array}$ & $\begin{array}{l}\text { Semana } \\
2\end{array}$ & \begin{tabular}{|c|} 
Semana \\
3
\end{tabular} & $\begin{array}{l}\text { Semana } \\
4\end{array}$ & $\begin{array}{l}\text { Semana } \\
5\end{array}$ & Total \\
\hline $\begin{array}{l}\text { Situación de centros } \\
\text { y servicios }\end{array}$ & $\begin{array}{c}6 \\
8,3 \%\end{array}$ & $\begin{array}{c}4 \\
5,6 \%\end{array}$ & $\begin{array}{c}1 \\
1,4 \%\end{array}$ & $2 \%$ & $\begin{array}{c}2 \\
2,8 \%\end{array}$ & $\begin{array}{c}15 \\
20,8 \%\end{array}$ \\
\hline $\begin{array}{c}\text { Recursos orientados } \\
\text { a las familias }\end{array}$ & $\begin{array}{c}1 \\
1,4 \%\end{array}$ & $\begin{array}{c}12 \\
16,7 \%\end{array}$ & $\begin{array}{c}8 \% \\
11,1 \%\end{array}$ & $\begin{array}{c}3 \\
4,2 \%\end{array}$ & $\begin{array}{c}2 \\
2,8 \%\end{array}$ & $\begin{array}{c}26 \\
36,1 \%\end{array}$ \\
\hline $\begin{array}{l}\text { Recursos orientados } \\
\text { a los profesionales }\end{array}$ & $\begin{array}{c}1 \\
1,4 \%\end{array}$ & $\begin{array}{c}4 \\
5,6 \%\end{array}$ & $\begin{array}{c}1 \% \\
1,4 \% \\
\end{array}$ & $\begin{array}{c}1 \% * \\
1,4 \%\end{array}$ & $\begin{array}{c}2 \\
2,8 \%\end{array}$ & $\begin{array}{c}9 \\
12,5 \%\end{array}$ \\
\hline Orientado a la formación & $\begin{array}{c}1 \\
1,4 \% \\
\end{array}$ & $\begin{array}{c}5 \\
6,9 \% \\
\end{array}$ & \begin{tabular}{|c|}
8 \\
$11,1 \%$ \\
\end{tabular} & $\begin{array}{c}3 \\
4,2 \% \\
\end{array}$ & $\begin{array}{c}4 \\
5,6 \% \\
\end{array}$ & $\begin{array}{c}21 \\
29,2 \% \\
\end{array}$ \\
\hline Plataformas & $\begin{array}{c}1 \\
1,4 \% \\
\end{array}$ & $\begin{array}{c}1 \\
1,4 \% \\
\end{array}$ & 0 & 0 & 0 & $\begin{array}{c}2 \\
2,8 \% \\
\end{array}$ \\
\hline TOTAL & $\begin{array}{c}10 \\
13,9 \% \\
\end{array}$ & $\begin{array}{c}26 \\
36,1 \% \\
\end{array}$ & $\begin{array}{c}18 \\
25 \% \\
\end{array}$ & $\begin{array}{c}9 \\
12,5 \% \\
\end{array}$ & $\begin{array}{c}10 \\
13,9 \%\end{array}$ & 73 \\
\hline
\end{tabular}

* Un recurso es a la vez de utilidad para familias y profesionales. Fuente: Elaboración propia.

Los recursos más orientados hacia la familia se concentraron fundamentalmente entre la segunda y tercera semana. Allí se contabilizan 20 de los 26 recursos de este tipo (el 76,9 \%). Por último, aquellos orientados a la formación de los profesionales comienzan a aparecer en la segunda semana y se distribuyen de forma algo más equitativa entre esta segunda semana y las siguientes tres semanas, hasta el final del periodo analizado.

Es de señalar que diversos recursos, como las videoconferencias de Plena inclusión sobre teleintervención en Atención Temprana, por ejemplo, continuaron durante 7 semanas, hasta el 13 de mayo. Fruto de ello se publicó una Guía de Teleintervención el 6 de mayo (Plena inclusión, 2020).

Desde la perspectiva del paradigma de intervención con el que se alinean, el 52,8 \% de los recursos no lo hace con ninguno específico (38 de los 72 recursos identificados). Por contra, un 45,8 \% de ellos están alineados con el paradigma de intervención 
centrado en la familia (33 de los 72 identificados). Solo un recurso (1,4\% del total) lo hace más con una intervención ambulatoria centrada en el niño, hablando de trabajar objetivos concretos en áreas específicas enviando, vía email, a la familia recomendaciones concretas sobre lo que tienen que hacer.

Por último, desde la perspectiva de la autoría o responsables de los recursos generados, destacan, por su volumen de aportaciones, 5 entidades diferentes. Los detalles de su distribución se resumen en la Tabla 4, donde se ha calculado el porcentaje que suponen sus aportaciones sobre los 72 recursos identificados.

\begin{tabular}{|c|c|c|c|c|c|c|}
\hline $\begin{array}{c}\text { TABLA 4. Distribución (frecuencia y porcentajes) de los recursos generados por las } \\
\text { entidades más activas durante el periodo analizado }\end{array}$ \\
\hline & & \multicolumn{5}{c|}{ Distribución por semana } \\
\hline Entidad & Recursos & 1 & 2 & 3 & 4 & 5 \\
\hline AEIPI & 4 docs; 3 vídeos; 3 apps & 2 & 4 & 1 & 0 & 3 \\
\hline AVAPACE & 8 vídeos & 0 & 4 & 2 & 2 & 0 \\
\hline Plena inclusión & 1 doc; 5 vídeos; 1 app & 1 & 2 & 2 & 1 & 1 \\
\hline Doble Equipo & 5 vídeos & 1 & 2 & 2 & 0 & 0 \\
\hline ACAIT / Equipo SIDI & 5 vídeos & 1 & 0 & 2 & 1 & 1 \\
\hline
\end{tabular}

Fuente: Elaboración propia.

Durante las dos primeras semanas de confinamiento se crearon dos aplicaciones específicas para atender dudas y facilitar recursos a familias y profesionales. En la primera semana, Plena inclusión lanzó una aplicación para móviles y tabletas bajo el título 'Frente al aislamiento. Nos conectamos' (entrada número 13 de la Tabla 1). Con esta aplicación se facilitaba el acceso a documentos, foros para preguntar dudas y agenda de actividades online. En la segunda semana, AEIPI dispuso otra aplicación, esta vez a través de la plataforma Twist (entrada número 23 de la Tabla 1), para poder generar un punto de encuentro y poder compartir los recursos que se estaban generando vía online.

\subsection{Análisis de ideas expresadas por los profesionales de Atención Temprana}

En relación con la formación y documentación multimedia que se ha ido generando, las ideas reflejadas por los profesionales hacen referencia a:

- Sensación de estar desbordados por la cantidad de material distribuido.

- Sentimiento de querer aportar más, tanto a familias como a otros profesionales, pero no poder llegar por falta de tiempo.

- Agradecimiento por contar con toda esa información y las posibilidades que abre.

- Agradecimiento a los esfuerzos de las distintas entidades por la documentación y formación que van facilitando. 


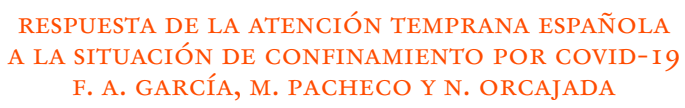

- Resulta reconfortante comprobar que hay mucha gente preocupada por los mismos temas y buscando soluciones para desarrollar la intervención a distancia y seguir apoyando a las familias.

- Sensación de estar ampliando la red de contactos profesionales, contribuyendo a favorecer la transdisciplinariedad y la coordinación.

En relación con las necesidades detectadas en las familias y las experiencias de interacciones vividas con ellas, los profesionales hablan de:

- Necesidad de dotar a las familias de recursos tecnológicos. No todas tienen posibilidad de videoconferencia: faltan medios o, incluso, acceso a internet.

- Gran flexibilidad, por parte del profesional, para adaptarse a las posibilidades tecnológicas de la familia. No es él quien decide la herramienta a utilizar para la teleintervención, sino la familia y sus recursos.

- Para algunos profesionales el contacto por teleintervención está contribuyendo a hacer aún más fuerte el vínculo profesional-familia, en ambas direcciones.

- Mayor necesidad de abordar y gestionar adecuadamente las emociones. Además, hacerlo de forma directa, identificándolas y reflexionando sobre sus causas y consecuencias.

- Las primeras semanas:

- Las familias no quieren nada especial. Están nerviosas, con mucha incertidumbre y con pocas ganas de plantearse actuaciones con el niño.

- Están muy agobiadas con la situación general que tienen en casa y lo último que quieren y necesitan es pensar en objetivos.

- Al llamarlas se las nota receptivas. Es un momento de desahogo, de sentirse comprendidas. Se alegran de que te acuerdes de ellas y de saber que tienen un apoyo.

- Se va entendiendo lo que las familias necesitan en este primer momento:

- No es momento de presionarlas con ideas, estrategias, ni demasiadas llamadas que les hagan pensar que no están trabajando lo suficiente. Inicialmente se tendía a ello, por el deseo del profesional de hacer y de ser útil. Pero el exceso de atención e información también las aturde.

- Lo primero es una llamada para que las familias sepan que los profesionales están allí, disponibles para ayudarles. Hacerles saber que pueden contactar cuando lo necesiten o quieran.

- Es importante descargarles presión. Que vean a los profesionales seguros de que los niños no van a parar su evolución, ni "empeorar" por estas semanas.

- Transmitir a las familias que todo el contacto y cariño que dan a sus hijos es ya un importante estímulo positivo.

- Después de las primeras semanas:

- Algunas familias contactan y demandan la continuidad de la intervención. Otras acuerdan esta continuidad aprovechando una segunda o tercera llamada por parte del profesional. 
- La primera parte de las primeras sesiones de teleintervención se dedican a la toma de contacto con la herramienta a utilizar. A explorar sus posibilidades, tanto por parte de profesionales como familias.

- Las familias han ido adaptándose a la situación de confinamiento. Aceptada la situación, presentan menos estrés y van ajustando sus nuevas rutinas.

- Están más abiertas y dispuestas a escuchar al profesional y colaborar con él planteándose objetivos funcionales ajustados a la nueva realidad.

- Se perciben mayores esfuerzos, por parte de la familia, para que el profesional entienda bien la situación que están viviendo y pueda brindarles la ayuda que necesitan.

- Aparecen familias que solicitan, por primera vez, los servicios de Atención Temprana. Esto hace que el profesional no solo deba adaptar el proceso de intervención a la telepráctica, sino, también, el proceso de valoración del niño y la familia. Se ve obligado a hacer una valoración más funcional e implicando a la familia en mayor medida.

- En las familias nuevas se observan comportamientos similares a los que ocurren en las visitas presenciales a las casas: durante las primeras sesiones, la familia controla su aspecto y su ubicación para la teleintervención, eligiendo un lugar de su casa tranquilo y perfectamente ordenado. Más tarde, no cuidan tanto esa imagen: se conectan con una indumentaria mucho más cómoda y en cualquier lugar de la casa, incluso mientras realizan la rutina que toca.

- Una profesional que combinaba en su horario familias en régimen ambulatorio y otras a las que visitaba en su domicilio para una intervención centrada en la familia comenta la sensación de que, al hablar con las familias de ambulatorio y explicarles lo que puede dar de sí una rutina diaria, se quedan muy tranquilas. Aceptan su realidad con más facilidad y están más receptivas al cambio, dada la situación de confinamiento que están viviendo.

- Los profesionales detectan en las familias capacidades que no esperaban. Al tener más tiempo para estar con sus hijos se organizan mejor, tienen interacciones más positivas con ellos y todo ello repercute en beneficio del menor y de la calidad de vida familiar. Hay familias que comentan conocer mucho mejor a sus hijos a partir de la situación de confinamiento.

- Se da valor al trabajo que siempre se viene haciendo con las familias desde Atención Temprana, incluso cuando se lleva a cabo una intervención ambulatoria. La poca o mucha competencia que la familia haya ido ganando en sus interacciones con los profesionales ahora está sumando para que muchas familias estén resolviendo bien la situación que enfrentan.

- Muchas familias se sienten desbordadas por la suma de tareas que reciben, del colegio, las propuestas que reciben desde Atención Temprana, teletrabajo, otros miembros de la familia, etc.

- Las tareas que envían algunos profesionales habituados a una intervención centrada en el niño se unen a las que se envían desde los colegios, al propio teletrabajo de los padres, etc. 
- Desde una intervención centrada en la familia, se entiende importante transmitir a las familias que el profesional no está, ni contacta, para darles más carga de trabajo de la que tienen. Su misión es ayudarles a encontrar las mejores estrategias para contribuir a la evolución del niño desde sus rutinas habituales y, en la situación de confinamiento que estamos viviendo, sobrellevar la carga de trabajo que pudieran suponer las necesidades especiales de su niño.

- Necesidad de transmitir tranquilidad y serenidad. A la vez, ofrecer recomendaciones de organización del tiempo que tienen ahora las familias para disfrutar con sus hijos. Precisamente esas recomendaciones son demandadas directamente por muchas familias. Es el momento de aprovechar y poner en valor oportunidades existentes en el entorno natural para compartir más con los hijos.

- Necesidad de cambiar el enfoque: mirar más hacia las necesidades de la familia y menos hacia las necesidades del niño.

- Necesidad de favorecer que los padres desvíen su foco de atención hacia otros miembros de la familia (hermanos, por ejemplo), relativizando la importancia de la discapacidad.

En relación con los problemas detectados a la hora de desarrollar la teleintervención, las ideas reflejadas por los profesionales hacen referencia a:

- Durante los primeros contactos, indecisión sobre la herramienta o aplicación más idónea para realizar la teleintervención.

- Dudas sobre cómo plantear la continuidad de la intervención.

- A pesar de las videoconferencias no siempre puedes ver todo lo que quisieras o no puedes ver, de forma adecuada, lo que está ocurriendo.

- El contacto telemático genera una dificultad añadida para saber si estás llegando realmente a la familia. El profesional necesita más tiempo para tener la seguridad de que la familia ha comprendido. Se ve obligado a ajustar más su discurso. A adaptar el lenguaje y las orientaciones a las posibilidades, características y situaciones reales de cada familia. Al no haber un contacto directo, se pierde mucha información que nos da la comunicación no verbal. Por ello, debe preocuparse, incluso más que en una intervención habitual, en asegurar que:

- La familia entiende al profesional, que comprenden el mensaje.

- El profesional está atendiendo a necesidades actuales reales de la familia y no a su interpretación de ellas.

- El profesional favorece la reflexión, para potenciar el aprendizaje y el desarrollo de competencias del cuidador principal.

- Aumenta el número de vídeos que la familia comparte con el profesional. Las familias sienten la necesidad de compartir esos vídeos para que el profesional pueda ver la situación en la que necesitan ayuda. Los profesionales que no se atrevían a pedir esas grabaciones se sienten, ahora, más seguros para hacerlo.

- La mayor disponibilidad de vídeos facilitados por las familias conduce a la necesidad de aprender a sacar más partido de su análisis. 
- Necesidad de herramientas que permitan compartir, entre familia y profesional, el visionado de los vídeos grabados, para facilitar una reflexión conjunta.

- Detección de carencias tecnológicas:

- Necesidad de disponer de un móvil o dispositivo de empresa para llevar a cabo la intervención. Los centros han invertido muchos recursos en dotar de material sus salas, pero muchos no cuentan con un móvil de empresa que facilite ahora la intervención o para que la familia pueda compartir fotos o vídeos de actividades cotidianas.

- Algunos profesionales carecen incluso de dirección de correo electrónico asociado a su puesto de trabajo.

- Mayoritariamente, los profesionales tienen que llevar a cabo la teleintervención a partir de sus propios recursos tecnológicos.

- Necesidad de conocer distintas herramientas y plataformas para mantener el contacto con las familias. Necesidad de formarse en esas herramientas. Un mismo profesional puede utilizar videollamada, WhatsApp, videoconferencia, etc., en función de las familias y sus posibilidades.

- Se detectan diferencias en el modo de hacer, según el paradigma de intervención en el que venía trabajando el profesional:

- Los profesionales acostumbrados a una intervención ambulatoria, centrada en el niño, tienen tendencia a mandar información a las familias, pautas de actuación y tareas a desarrollar en el entorno.

- Los profesionales acostumbrados a una intervención en el entorno, centrada en la familia, tienen más facilidad para escuchar las nuevas necesidades de las familias y ayudarlas a encontrar y ajustar nuevas estrategias para esas nuevas realidades.

- Algunos profesionales que intervienen desde una práctica ambulatoria empiezan a entender el sentido de la intervención centrada en la familia. Sienten que les faltan herramientas para realmente llegar a comprender sus necesidades y comunicarse de forma eficaz con ellas, para conseguir ser un verdadero apoyo efectivo en la nueva situación. Esto los lleva a querer empezar su proceso de transformación hacia las prácticas de intervención centradas en la familia.

- Una vez que las familias se van adaptando a la situación de confinamiento se van acostumbrando, también, a la teleintervención. Algunas se van haciendo más exigentes, demandan más contacto, envían más vídeos... El profesional tiene que retomar cierto control y establecer horarios de atención.

- Los profesionales entienden que las familias han estado siendo atendidas durante el confinamiento.

Otras cuestiones que aparecen en los comentarios de los profesionales:

- Incertidumbre inicial sobre la continuidad o no del trabajo, especialmente en los equipos habituados a una intervención ambulatoria centrada en el niño. Algunos no ven la posibilidad de continuar y ven la solución en el cierre institucional y el acogimiento a las medidas de ERTE. 
- Esta incertidumbre se entremezcla, en muchos profesionales, con el deseo de seguir siendo un apoyo para las familias a través de fórmulas de teleintervención.

- Dudas iniciales sobre la interpretación exacta y las consecuencias de las normas que se van estableciendo en relación con el mundo de la discapacidad: posibilidad de salida o no a la calle, de llevar o no la mascarilla...

- Necesidad de reorganizar y distribuir el tiempo disponible de otra manera y, sobre todo, cumplir con los tiempos previstos para cada tarea, para poder llegar a cumplir con todo lo previsto (intervención con la familia, formación, consulta de recursos generados, etc.).

- Necesidad de registrar el teletrabajo que se está realizando: llamadas o videollamadas realizadas, registros de WhatsApp, documentación aportada...

- Conveniencia de hacer alguna encuesta entre las familias para que ellas valoren el teletrabajo realizado desde Atención Temprana.

4.3. Registro de opiniones, sensaciones y sentimientos expresados por profesionales de Atención Temprana ante el planteamiento de vuelta a una "nueva normalidad"

- Los profesionales que han encontrado un buen ajuste en el teletrabajo con las familias ven contradictorio abandonar la teleintervención, con los resultados que se están obteniendo y con el esfuerzo de formación realizado por instituciones como Plena inclusión. Otros son más partidarios de volver a un trabajo presencial.

- Temen tanto la vuelta a los domicilios como a la intervención ambulatoria. Ven difícil evitar el contacto y el posible contagio. Se plantean las enormes dificultades para asegurar medidas de higiene y distanciamiento social, debido a:

- El trabajo directo con niños que tosen, babean, chupan los objetos...

- Posibles problemas de higiene en domicilios a visitar y dificultades para adoptar medidas de higiene en los desplazamientos a domicilios, para una intervención centrada en la familia y desarrollada en entornos naturales.

- Se ve más viable asegurar medidas de higiene en una intervención en locales del centro de Atención Temprana, especialmente si la interacción se lleva a cabo con las familias y no tanto con los niños. El profesional puede estar más preparado para desinfectar su entorno controlado antes de la intervención con otra familia.

- Si Atención Temprana es un recurso esencial que puede hacerse por teleintervención, como se ha venido demostrando, ¿por qué generar situaciones de riesgo, en una población especialmente sensible, volviendo a un régimen ambulatorio o de visitas a los domicilios?

- Algunos profesionales se plantean problemas de responsabilidad moral en caso de contagiar involuntariamente a un niño o su familia. Miedo al contagio y a ser fuente de contagio. 
- Los profesionales ven familias muy despreocupadas, como que ya no pasa nada; y otras que ven una locura volver a una intervención presencial.

- Algunas familias pueden querer volver a una intervención ambulatoria por el mero hecho de salir y volver a cierta rutina. Por respiro. Sobre todo, casos de niños con problemas de conducta que siempre han trabajado bajo una modalidad de intervención ambulatoria centrada en el niño.

\section{Discusión y conclusiones}

En este trabajo se han revisado recursos generados en torno a la Atención Temprana, durante las cinco primeras semanas de confinamiento, así como opiniones, sensaciones y sentimientos compartidos por profesionales de la disciplina. La visión de estos profesionales ante la situación vivida, reflejada en este trabajo, complementa la visión de las familias recogida en otros trabajos (Martín-Tarrascón y Segarra, 2020; Neece et al., 2020; UCCAP y ACAP, 2020; Willner et al., 2020).

El volumen de documentación multimedia sobre Atención Temprana generado ha sido francamente asombroso. Superando, en mucho, la documentación de este tipo que se venía generando, incluso sumando los doce meses anteriores. Los propios profesionales señalan haberse sentido desbordados por su cantidad. Al principio predominaba la información sobre la situación en que estaba la Atención Temprana. Después los esfuerzos se volcaron en generar recursos orientados a profesionales y familias.

Este volumen de trabajo y esfuerzo, totalmente altruista, ha implicado a múltiples profesionales y asociaciones, liderados por entidades como AEIPI, AVAPACE, Equipo SIDI y Plena inclusión. Refleja la buena salud de la Atención Temprana en España y la calidad y valía de su tejido profesional. Profesionales y familias han podido encontrar, en la documentación que se iba generando y depositando en internet, nuevas ideas y fórmulas imaginativas para seguir apoyando y ayudando al niño y a su familia.

Toda esa iniciativa se ha desarrollado mientras estábamos inmersos en la situación de confinamiento. Una situación que no era fácil para nadie. Pero los numerosos profesionales implicados compatibilizaron sus acciones altruistas de formación online con las sesiones de teleintervención con las familias que trabajaban o sus otras ocupaciones laborales y familiares. Una vez más, y a pesar de las circunstancias, queda reflejado el nivel de implicación de los profesionales dedicados a esta disciplina.

Se han analizado también ideas y respuestas de los profesionales ante las nuevas necesidades de las familias y de la propia teleintervención. A pesar de las dificultades encontradas para dar continuidad a la intervención, los profesionales entienden que las familias han seguido siendo atendidas, puesto que no han dejado de mantener contacto con ellas y se ha intentado dar respuesta a sus necesidades. Esta conclusión se ve avalada por las opiniones expresadas por las propias familias en estudios ya publicados (Martín-Tarrascón y Segarra, 2020; Neece et al., 2020; UCCAP y ACAP, 2020). Las necesidades atendidas se centraron, sobre todo inicialmente, en ayudar a 
la adaptación a la situación de confinamiento; a reorganizar sus nuevos horarios y rutinas; y en ofrecer un importante apoyo emocional, mostrando, si cabe, una mayor capacidad de escucha. Para dar este apoyo y ayuda, los profesionales no solo han dedicado tiempo y esfuerzo personal, sino también sus propios recursos técnicos, ante la falta de teléfonos con números de empresa e, incluso, de cuentas de correo electrónico asociadas a su trabajo.

Ante los cambios en la intervención a que obligaba el confinamiento, los profesionales tenían puntos de partida claramente diferentes. Reflejo, sin duda, de la situación de convivencia de dos paradigmas de intervención en nuestro país (Escorcia et al., 2016; García-Grau et al., 2019; García-Sánchez et al., 2014; Gracia et al., 2019). Un volumen importante de profesionales seguían habituados a una práctica de intervención ambulatoria, centrada en las necesidades del niño. Acostumbraban a intervenir directamente sobre él, para luego dar orientaciones a la familia sobre actuaciones a realizar en la casa, muchas veces pensadas como apoyo a esa intervención realizada con base en objetivos profesionales. Muchos de ellos inicialmente vieron, en el confinamiento, la necesidad evidente de interrumpir la intervención, por el alto riesgo de contagio que implicarían los desplazamientos y la intervención sobre el niño. Parecía imposible pensar en una intervención a distancia. En base en ello, en más de un servicio se llegó a plantear el cierre total y la aplicación de ERTES a los profesionales. Sin embargo, estos profesionales siempre venían realizando, además de su intervención directa sobre el niño, un acompañamiento a las familias y un asesoramiento sobre esas posibilidades de dar continuidad a la intervención en el hogar. Tras algunos días de desconcierto empezó a pensarse que esa parte de la intervención podía y debía seguir realizándose a distancia.

Por el contrario, la reacción de continuidad del servicio en los profesionales que venían llevando a cabo una intervención centrada en la familia y desarrollada en los entornos naturales fue más inmediata y espontánea. Estaban ya acostumbrados a atender las preocupaciones de la familia en el entorno, para apoyarles en la identificación y ajuste de sus propias estrategias para mejorar la actividad y participación del niño y, con ello, la calidad de vida familiar. Inmediatamente entendieron que la situación de confinamiento generaría nuevas preocupaciones y necesidades y que había que seguir dando un apoyo efectivo, basado en la participación activa de los cuidadores principales del menor. Para muchos de ellos, no sería la primera vez que respondían a demandas de las familias por teléfono. También estaban ya habituados a recibir vídeos con los que evaluar una situación y ayudar a reflexionar sobre ella y a encontrar estrategias para abordarla. Nada impedía seguir haciendo y generalizando estas tareas, que ya eran un complemento habitual de la intervención. La única dificultad, en este sentido, sería encontrar la manera de ver y analizar esos vídeos conjuntamente en la distancia.

Con las primeras experiencias de teleintervención, los profesionales habituados a una práctica ambulatoria pronto fueron entendiendo que el apoyo que podían dar a las familias no podía consistir en intentar que los padres reprodujeran las intervenciones que el profesional pudiera venir haciendo con el niño. Su tendencia inicial fue en ese sentido, asesorando y orientando sobre ejercicios a realizar, así como facilitando documentación y programaciones con objetivos profesionales. Pero esas orientaciones se 
sumaban a las que ya estaban recibiendo las familias desde el campo educativo, generándoles más estrés y terminando por ser rechazadas. Por ello, el esfuerzo se fue dirigiendo hacia estrategias de control de conducta y potenciar autonomía y participación del niño, alejándose de objetivos de desarrollo del currículum escolar, que ya estaban siendo atendidos.

En esa nueva tarea encontraban carencia de herramientas y estrategias prácticas para potenciar, en las familias, sus propias competencias; capacitarlas y apoyarlas para que desarrollasen sus propias iniciativas que contribuyeran al aprendizaje y al bienestar del niño en las rutinas diarias. Justo las herramientas que sus compañeros, que ya desarrollaban prácticas centradas en la familia, ya implementaban. Por ello, no sorprende que un volumen muy importante de los recursos desarrollados y compartidos estuvieran alineados con este paradigma de intervención. Eran los recursos que mejor podían atender las nuevas necesidades que presentaban las familias.

El reto para los profesionales implicados ya con prácticas de intervención centradas en la familia era seguir haciendo lo mismo a través de la teleintervención. El reto para sus compañeros, no iniciados en esas prácticas, implicaba, además, cambiar de forma importante el objetivo de la intervención. El alineamiento de los recursos que se fueron generando con el paradigma de prácticas centradas en la familia respondía a las necesidades de unos y otros profesionales. Se trataba de encontrar cómo, a través de la teleintervención, seguir competenciando a las familias y ajustando estrategias para que aprovecharan oportunidades de aprendizaje en el entorno natural y mejoraran su calidad de vida familiar en sus rutinas habituales.

En cuanto a las familias, durante el confinamiento tenían que aceptar que por vía telemática podían recibir suficiente apoyo del profesional para poder atender las necesidades del niño y de la propia familia. A la vez, tenían que aceptar que el profesional no podía actuar directamente sobre el problema del niño. En este doble desafío, las familias que venían recibiendo una intervención centrada en la familia partían, también, con una clara ventaja. Ya estaban acostumbradas a que no es el profesional, sino los propios padres, la figura que lleva a cabo la intervención en el entorno que ayuda al niño a progresar en sus aprendizajes y en su desarrollo. Sus profesionales de referencia ya venían trabajando, en colaboración con ellas, centrando el trabajo en hacerlas sentirse y ser cada vez más competentes, realmente capaces de atender las necesidades de sus hijos. Por eso, para esas familias, buena parte del camino estaba andado. La situación de confinamiento, con todo lo excepcional que conllevaba, simplemente generaba cambios, ciertamente a veces muy sustanciales, en el entorno, en las rutinas diarias y en la disponibilidad de los apoyos habituales (abuelos, amigos) que desaparecieron completamente. Cambios a los que había que ajustarse, encontrando nuevas estrategias para mantener cierta calidad de vida familiar y aprovechar las nuevas oportunidades de aprendizaje, que también aparecían. Así, para estas familias, el mayor cambio era llevar a cabo de forma telemática las sesiones de intervención que ya venían realizando. Por tanto, notaron mucho menos el cambio y aceptaron rápido la situación para seguir trabajando. Pasaron a plantearse nuevos objetivos y estrategias ajustados a la nueva situación. Algunos padres incluso veían más posibilidades 
de ayudar a sus hijos, al tener, durante el confinamiento, más situaciones para experimentar y apoyarlos en su aprendizaje.

Por el contrario, las familias no habituadas a una intervención centrada en ellas no terminaban de entender cómo podrían continuar apoyando a sus hijos. Rechazaban la idea de la teleintervención, pensando que eso supondría más carga de trabajo para ellas, ya que suponía 'ponerse ellas a trabajar'. A ello contribuían, al menos inicialmente, los mensajes que recibían de los terapeutas de su hijo, cuando estos venían orientados por objetivos profesionales. A la vez, su dependencia de la asistencia al centro y de la sesión de intervención de los profesionales con el niño les hacía sentir que sus hijos estaban perdiendo la oportunidad de seguir avanzando. Esta sensación se tradujo, más adelante, en presión sobre las administraciones y sobre los equipos para volver a una atención presencial. Todo ello a pesar de que las propias familias han observado un porcentaje mayor de evolución que de regresión en sus hijos, aunque hayan aparecido nuevas dificultades en el comportamiento, sueño y alimentación, provocadas quizá por el cambio radical de rutinas que implicaba el confinamiento (Martín-Tarrascón y Segarra, 2020; Neece et al., 2020; UCCAP y ACAP, 2020).

Pensando en esa vuelta a la "nueva normalidad", se sigue detectando cierta diferencia en los dos colectivos de profesionales descritos, con base en el paradigma de intervención que vienen implementando. Los profesionales habituados a la implementación de prácticas centradas en la familia ven contradictorio el mensaje de abandonar la teleintervención con el esfuerzo realizado desde instituciones como Plena inclusión para fomentarla. Consideran que el abandono de la teleintervención supone dificultades para controlar las medidas de higiene recomendadas y evitar posibles contagios en las visitas a los domicilios. Por ello, algunos profesionales se plantean, incluso, reanudar la intervención centrada en la familia, pero desarrollándola en el centro. Por su parte, los profesionales que siguen habituados a una intervención centrada en el niño ven más urgente y necesaria la vuelta a una intervención presencial, para retomar su rol en la intervención directa con el niño.

También pensando en esa vuelta a la 'normalidad' encontramos opiniones de profesionales que recuerdan las características de los usuarios atendidos en Atención Temprana. Se trata de una población vulnerable, cuyo riesgo de complicaciones, ante el contagio por coronavirus, se considera elevado. Esto hace que los profesionales sientan una gran responsabilidad, llevándolos a discutir y analizar responsabilidades éticas en caso de un posible contagio. Entienden necesario valorar no solo las consecuencias personales en caso de contraer la infección, sino el cargo de conciencia que les supondría el hecho de poder contagiar a niños y familias con las que trabajan. La exposición a este riesgo hace que se sume una nueva carga emocional a la que normalmente ya soportan estos profesionales al intervenir con las familias. Por tanto, la gestión de emociones, en este sentido, es algo necesario de abordar en sesiones de supervisión reflexiva, llevadas a cabo con los equipos profesionales.

Es posible que la situación vivida durante los meses de confinamiento haya supuesto un antes y un después en la Atención Temprana que conocíamos. Por un lado, todas las iniciativas profesionales y el uso intensivo de las diferentes herramientas que han permitido la teleintervención han supuesto una revolución digital para todos y, 
posiblemente también, para la disciplina. Incluso cabe plantearse la necesidad de reasignación de recursos, cuando hemos vivido la importancia de las herramientas para la teleintervención, el manejo de vídeos y su custodia, etc. Se hace necesario pensar en generalizar la disponibilidad de móviles de empresa, por ejemplo, y facilitar, a las familias que lo requieran, los medios mínimos necesarios para que puedan compartir fotos o vídeos para visionar actividades cotidianas. Especialmente, conforme vamos siendo conscientes de que la 'nueva normalidad' va a convivir con nosotros más tiempo del que todos desearíamos y nos exige, por responsabilidad, potenciar la teleintervención (Poole et al., 2020).

La teleintervención en Atención Temprana no es algo totalmente nuevo. A nivel internacional existen experiencias que han demostrado su eficacia para atender necesidades de familias en ámbitos rurales y en problemáticas concretas como autismo o déficits auditivos (Havenga et al., 2017; McCarthy et al., 2010; Meadan et al., 2013; Olsen et al., 2012). Y en prácticas de intervención centradas en la familia lleva tiempo siendo habitual el intercambio de grabaciones en vídeo y de información sobre problemas puntuales a través de WhatsApp, correos electrónicos y llamadas telefónicas. La experiencia de confinamiento vivida y la necesidad de recurrir a la teleintervención ha servido para acelerar las reflexiones sobre el mejor protocolo de uso de las diferentes posibilidades tecnológicas para la teleintervención en Atención Temprana (Plena inclusión, 2020; Poole et al., 2020).

Por otro lado, la utilidad constatada de los recursos telemáticos para la formación continua del profesional nos hace plantearnos el uso de estos recursos en las futuras acciones formativas y reuniones de intercambio de conocimientos. Estos recursos han facilitado el acceso a profesionales de muy distintas zonas geográficas, reducen costes y tiempo, evitan desplazamientos y vienen consiguiendo prácticamente los mismos objetivos y el mismo nivel de satisfacción que si se hubiesen impartido de forma presencial.

La situación por la que hemos atravesado debe contribuir a que tanto profesionales como familias entiendan la necesidad de que la familia sea parte del equipo y de la intervención de manera más proactiva. No podemos dejar que las familias sigan siendo dependientes del profesional, porque el profesional no puede acompañarlos durante toda su vida. Siempre habrá situaciones en las que las familias se verán solas y deben saber afrontarlas. Por este motivo, el objetivo del profesional de Atención Temprana debe pasar por hacer competente a la familia, para que esta pueda tomar sus propias decisiones y favorecer el aprendizaje de su hijo, sin ningún tipo de temor, cuando el profesional no esté. En este sentido, durante la experiencia de confinamiento vivida, la red de profesionales de Atención Temprana, independientemente del paradigma bajo el que desempeñe su labor, puede que haya modificado positivamente la forma en la que venía realizando las intervenciones. Sería deseable que estos aprendizajes y nuevas prácticas adquiridas perduraran en el tiempo y animamos a que así sea.

Concluimos, de todo lo expuesto, que la Atención Temprana goza de buena salud en nuestro país, aunque sigamos padeciendo carencias importantes, como la falta de un marco legislativo acorde con las necesidades reales de la disciplina. Al convencimiento que ya tenían todos los profesionales sobre la importancia de la 
familia, se une ahora el impulso de un colectivo profesional cada vez mayor, que ha entendido los principios rectores de unas prácticas de intervención centradas en esas familias. Un colectivo profesional que cada vez va teniendo más claro cómo llegar a las familias, cómo atender sus verdaderas necesidades, mejorando su calidad de vida, la calidad de las interacciones con el niño, y su competencia para favorecer su desarrollo dentro de sus posibilidades. Precisamente todo aquello que se recoge como Prácticas Recomendadas en Atención Temprana (DEC, 2014).

\section{Referencias bibliográficas}

Acebes, B. y Montanera, R. (2020). Estudio de Redes Sociales 2020. IAB.SPAIN/ELOGIA. Recuperado de https://iabspain.es/estudio/estudio-redes-sociales-2020/

Castellanos, P., García Sánchez, F. A. y Mendieta, P. (2000). La estimulación sensoriomotriz desde el Modelo Integral de Intervención en Atención Temprana. Siglo Cero, 31(4), $5-13$.

Castellanos, P., García-Sánchez, F. A., Mendieta, P., Gómez, L. y Rico, M. D. (2003). Intervención sobre la familia desde la figura del terapeuta-tutor del niño con necesidades de Atención Temprana. Siglo Cero, 34(3), 5-18.

Dec-Division for EARly ChILdhood. (2014). DEC recommended practices in early intervention/early childhood special education 2014. Council for Exceptional Children. Recuperado de http://www.dec-sped.org/recommendedpracticesDEC

Eggbeer, L., Mann, T. y Seibel, N. (2008). Reflective supervision: past, present, and future. Zero to Three, 28, 5-9.

Escorcia, C. T., García-Sánchez, F. A., Orcajada, N. y Sánchez-López, M. C. (2016). Perspectiva de las prácticas de atención temprana centradas en la familia desde la logopedia. Revista de Logopedia, Foniatría y Audiología, 36(4), 170-177. https://doi: 10.1016/j. rlfa.2016.07.002

García-Grau, P., Martínez-Rico, G., McWilliam, R. A. y Cañadas, M. (2019). Typical and ideal practices in Early Intervention in Spain during a transformation process of professional practices. Journal of Early Intervention, 42(1), 3-19. https://doi. org/10.1177/1053815119859046

García-SÁnchez, F. A. (2002a). Reflexiones acerca del futuro de la Atención Temprana desde un modelo integral de intervención. Siglo Cero, 32(2), 5-14.

García-SÁnchez, F. A. (2002b). Atención Temprana: elementos para el desarrollo de un Modelo Integral de Intervención. Bordón, 54(1), 39-52.

García-Sánchez, F. A. (2003). Objetivos de futuro de la Atención Temprana. Revista de Atención Temprana, 4, 32-37.

García-SÁnchez, F. A. (2017, 7 de junio). ASTRAPACE. Atención Temprana y Universidad: una colaboración para una intervención centrada en la familia. Comunicación presentada al Encuentro Autonómico de Buenas Prácticas. Plena inclusión Región de Murcia.

García-Sánchez, F. A., Escorcia, C. T., Sánchez-López, M. C., Orcajada, N. y HernánDez-Pérez, E. (2014). Atención Temprana centrada en la familia. Siglo Cero, 45(3), 6-27. Recuperado de https://sid.usal.es/20896/8-2-6

García-Sánchez, F. A. y Mendieta, P. (1998). Análisis del tratamiento integral de atención temprana. Revista de Atención Temprana, 1(1), 37-43. 
Gat (2000). Libro Blanco de la Atención Temprana. Real Patronato sobre Discapacidad.

GAT (2011). La realidad actual de la AT en España. Real Patronato sobre Discapacidad.

Giné, C., García-Dié, M. T., Gràcia, M. y Vilaseca, R. (2005). Early intervention in Spain: an overview of current practices. En M. GuRALnick (Ed.), The developmental systems approach to Early Intervention (pp. 543-570). Paul Brookes publishing.

Giné, C., Gràcia, M., Vilaseca, R. y Balcells, A. (2009). Trabajar con las familias en Atención Temprana. Revista Interuniversitaria de Formación del Profesorado, 65(23,2), 95-113.

Giné, C., Gràcia, M., Vilaseca, R. y García-Díe, M. T. (2006). Repensar la atención temprana: propuestas para un desarrollo futuro. Infancia y Aprendizaje, 29(3), 297-313.

Giné, C., Vilaseca, R., Gràcia, M. y García-Díe, M. T. (2004). Early Intervention in Spain. Infants and Young Children, 17(3), 247-257.

Gracia, M., Simón, C., Salvador-Beltrán, F., Alcocer, A. L. A., Mas, J. M., Giné, C. y Dalmau, M. (2019). The transition process from center-based programmes to familycentered practices in Spain: a multiple case study. Early Child Development and Care, O(0), 1-13. https://doi.org/10.1080/03004430.2018.1564916

Gútiez, P. (2010). Early Childhood intervention in Spain: standard needs and changes. International Journal of Early Childhood Special Education (INT-JECSE), 2(2), 136-148.

Havenga, E., Swanepoel, D. W., Le Roux, T. y Schmid, B. (2016). Tele-intervention for children with hearing loss: a comparative pilot study. Journal of Telemedicine and Telecare, 23(1), 116-125. https://doi.org/10.1177/1357633X15617886

Mackin-Brown, M. (2016). Reflective supervision. HIPPY USA National Leadership Conference.

Martín-Tarrascón, S. y Segarra, S. (2020). Experiencia de confinamiento en familias atendidas en el CDIAP del Maresme. Desenvolopa. La Revista d'Atenció Precoç, julio, 1-21. Recuperado de http://www.desenvolupa.net/Jornades-i-presentacions/Experiencia-deconfinamiento-en-familias-atendidas-en-el-CDIAP-del-Maresme-Sergio-Martin-Tarrason-Sabina-Segarra-Mateu-07-2020

McCarthy, M., Muñoz, K. y White, K. R. (2010). Teleintervention for infants and young children who are deaf or hard-of-hearing. Pediatrics, 126(Supplement 1), S52-S58. https:// doi.org/10.1542/peds.2010-0354J

Meadan, H., Meyer, L. E., Snodgrass, M. R. y Halle, J. W. (2017). Coaching parents of young children with autism in rural areas using internet-based technologies: a pilot program. $R u$ ral Special Education Quarterly, 32(3), 3-10. https://doi.org/10.1177/875687051303200302

Mendieta, P. y García Sánchez, F. A. (1998). Modelo integral de intervención en atención temprana: organización y coordinación de servicios. Siglo Cero, 29(4), 11-22.

Neece, C., McIntyre, L. L. y Fenning, R. (2020). Examining the impact of COVID-19 in ethnically diverse families with young children with intellectual and developmental disabilities. Journal of Intellectual Disability Research, 6, 1-11. https://doi.org/10.1111/jir.12769

Olsen, S., Fiechtl, B. y Rule, S. (2012). An evaluation of virtual home visits in Early Intervention: feasibility of “virtual intervention"”. The Volta Review, 112(3), 267-281.

PARlakian, R. (2001). Look, listen, and learn: reflective supervision and relationship-based work. ZERO TO THREE.

Plena inclusión (2020). Cómo implementar la teleintervención en Atención Temprana. Plena inclusión. Recuperado de https://www.plenainclusion.org/informate/publicaciones/ como-implementar-la-teleintervencion-en-la-atencion-temprana 
Poole, M. E., Fettig, A., McKee, R. A. y Gauvreau, A. N. (2020). Inside the virtual visit: using tele-intervention to support families in Early Intervention. Young Exceptional Children, 24(1), 109625062094806-12. https://doi.org/10.1177/1096250620948061

Real Decreto por el que se declara el estado de alarma para la gestión de la situación de crisis sanitaria ocasionada por el COVID-19. (Real Decreto 463/2020, 14 de marzo). Boletín Oficial del Estado, n. ${ }^{\circ}$ 67, 2020, 14 de marzo. Recuperado de https://www.boe.es/eli/es/ $\mathrm{rd} / 2020 / 03 / 14 / 463 / \mathrm{con}$

SÁnchez, J. M. (2020, 2 de abril). El uso de redes sociales en España aumenta un 55 \% en la pandemia de coronavirus. $A B C$. Recuperado de https://www.abc.es/tecnologia/redes/

Tudela, V., Rico, M. D. y García-Sánchez, F. A. (2017, 5 de septiembre). Apoyo y consulta reflexiva para el desarrollo de prácticas centradas en la familia. Contribución presentada al 2. ${ }^{\circ}$ Encuentro de Transformación de la Atención Temprana que queremos. Plena Inclusión, Madrid.

UCCAP y ACAP (2020). Informe i resultats: enquesta d'atenció als CDIAP durant el confinament. Recuperado de http://uccap.com/shared/uploads/pdf/cb4e1-informe-atencio-precoc-covid-v4.pdf

Willner, P., Rose, J., Stenfert Kroese, B., Murphy, G., Langdon, P., Clifford, C., HutChings, H., Watkins, A., Hiles, S. y Cooper, V. (2020). Effect of the covid-19 pandemic on the mental health of carers of people with intellectual disabilities. Journal of Applied Research in Intellectual Disabilities, jar.12811-26. https://doi.org/10.1111/jar.12811 